ARTICLE

https://doi.org/10.1038/s41467-018-07741-6

\title{
Lysosomal protease deficiency or substrate overload induces an oxidative-stress mediated STAT3-dependent pathway of lysosomal homeostasis
}

\author{
Jonathan Martínez-Fábregas', Alan Prescott', Sander van Kasteren (10 2 ${ }^{1}$, Deena Leslie Pedrioli ${ }^{3,7}$, \\ Irwin McLean (1) ${ }^{3}$, Anna Moles ${ }^{4,8}$, Thomas Reinheckel ${ }^{5}$, Valeria Poli ${ }^{6}{ }^{6} \&$ Colin Watts (i) ${ }^{1}$
}

Diverse cellular processes depend on the lysosomal protease system but how cells regulate lysosomal proteolytic capacity is only partly understood. We show here that cells can respond to protease/substrate imbalance in this compartment by de novo expression of multiple lysosomal hydrolases. This response, exemplified here either by loss of asparagine endopeptidase (AEP) or other lysosomal cysteine proteases, or by increased endocytic substrate load, is not dependent on the transcription factor EB (TFEB) but rather is triggered by STAT3 activation downstream of lysosomal oxidative stress. Similar lysosomal adaptations are seen in mice and cells expressing a constitutively active form of STAT3. Our results reveal how cells can increase lysosomal protease capacity under 'fed' rather than 'starved' conditions that activate the TFEB system. In addition, STAT3 activation due to lysosomal stress likely explains the hyperproliferative kidney disease and splenomegaly observed in AEP-deficient mice.

\footnotetext{
${ }^{1}$ Division of Cell Signalling and Immunology, School of Life Sciences, University of Dundee, Dundee DD1 5EH, UK. ${ }^{2}$ Division of Bio-Organic Chemistry, Leiden Institute of Chemistry, Einsteinweg 55, Leiden 2333CC, Netherlands. ${ }^{3}$ Division of Molecular Medicine, School of Life Sciences, University of Dundee, Dundee DD1 5EH, UK. ${ }^{4}$ Fibrosis Research Group, Institute of Cellular Medicine, Newcastle University, Newcastle upon Tyne NE2 4HH, UK. ${ }^{5}$ Institute of Molecular Medicine and Cell Research, Medical Faculty, Albert-Ludwigs-University, Freiburg D-79104, Germany. ${ }^{6}$ Department of Genetics, Biology and Biochemistry, University of Turin, Via Nizza 52, 10126 Turin, Italy. ${ }^{7}$ Present address: Department of Molecular Mechanisms of Disease, University of Zurich, Winterthurestrasse190 8057 Zurich, Switzerland. ${ }^{8}$ Present address: Institute of Biomedical Research of Barcelona, Spanish Research Council, Barcelona 08036, Spain. Correspondence and requests for materials should be addressed to J.M.-F. (email: j.martinezfabregas@dundee.ac.uk) or to C.W. (email: c.watts@dundee.ac.uk)
} 
E ndosomes and lysosomes are now known to participate in multiple aspects of cell and tissue physiology besides their classical role in degradation of endocytosed substrates. They host key signalling systems such as the nucleic acid sensing Tolllike receptors (TLRs) ${ }^{1,2}$ and mTOR pathway ${ }^{3,4}$, and they generate immunological information through class II MHC-mediated antigen presentation ${ }^{5,6}$. Lysosomal proteases can drive a physiologically important caspase-independent cell death pathway ${ }^{7,8}$, and lysosome-like organelles allow cytotoxic leucocytes such as CD8 T cells, mast cells and eosinophils to execute their specific functions ${ }^{9}$. In addition, lysosomes are central to autophagy ${ }^{10}$. Most of these functions depend on protease activities found in the lysosomal lumen. Because these hydrolytic events are separated from the major cellular signalling pathways by the lysosomal membrane until recently it has not been obvious how a requirement for more or less hydrolytic capacity would be signalled to the cytosol and onwards to the transcriptional apparatus.

How lysosomal gene expression is controlled was advanced substantially by the identification of a signalling pathway that leads to the activation of transcription factor EB (TFEB) ${ }^{11,12}$. In response to cellular starvation, some types of lysosomal stress and some lysosomal storage diseases, TFEB translocated from the cytosol to the nucleus to drive the transcription of a variety of genes involved in lysosomal and autophagic function ${ }^{3,13}$. TFEB is negatively regulated by sequestration in the cytosol but in response to nutrient deprivation and reduced mTORC1 activity it becomes dephosphorylated, enters the nucleus and activates its target genes. In addition, a PKC-dependent but mTORC1independent pathway for TFEB activation was recently described ${ }^{14}$. As important as this pathway is, there are reasons to suspect that additional regulatory mechanisms of lysosomal hydrolytic capacity may exist. For example, an increase in lysosomal protein substrate load and/or the accumulation of undegraded protein substrates is not expected to induce the TFEB pathway since this would be more consistent with a 'fed' rather than starved state. Nonetheless, increased hydrolytic capacity is likely needed to restore the status quo but how this would be achieved in the absence of mTOR/TFEB signalling is not clear.

Deletion of individual murine lysosomal proteases results in clear tissue-specific phenotypes illustrating that they have nonredundant functions ${ }^{15-18}$. Lysosomal proteolytic capacity relies mainly on three different enzyme families: the papain-like cysteine proteases (e.g. cathepsins B and L), the pepsin-related aspartyl proteases (cathepsins D and E) and a distinct cysteine protease known as asparaginyl endopeptidase (AEP) or legumain ${ }^{19-21}$. AEP shows high specificity for cleavage after asparagine ${ }^{19,20}$, suggesting it has specific processing functions. Consistent with this, AEP has been shown to make activating cleavages in TLR9 and TLR7 in dendritic cells ${ }^{1,22}$, and to participate in antigen processing 23 . AEP has also been recently linked to both exitoneurotoxicity and to neurofibrillary pathology through its site-specific cleavage of the DNAse inhibitor SET and tau, respectively ${ }^{24,25}$. Mice lacking AEP develop hyperproliferative kidney disease ${ }^{26}$ and several indications of hemophagocytic lymphohistiocytosis including hepatosplenomegaly 27 . How the absence of AEP triggers these hyperproliferative states is not known, but AEP is particularly abundant in the kidney proximal tubule ${ }^{19,26}$

We demonstrate here a TFEB-independent, STAT3-dependent signalling pathway for lysosomal protease homoeostasis triggered by loss of lysosomal cysteine proteases or substrate overload. Chronic or acute AEP-deficiency promotes STAT3-dependent transcription of all three lysosomal protease families including AEP itself as well as other hydrolases. Lysosomal oxidative stress appears to be the primary driver of Jak2-STAT3 activation which is also observed in the AEP-deficient kidney proximal tubular system and which may explain the hyperproliferative disease and loss of kidney function in AEP-null mice.

\section{Results}

The lysosome detects loss of AEP activity and responds by increasing expression of multiple proteases. Deletion of AEP in the murine kidney leads to proximal tubular cell (PTC) hyperplasia, interstitial fibrosis and eventually loss of kidney function ${ }^{26}$ At the morphological level lysosomes appeared more prominent in AEP-null kidney PTC and cathepsin D (CtsD) staining was more intense (Fig. 1a, b) indicating accumulation of undegraded material and/or increased expression of lysosomal proteins. Indeed, the levels of CtsL, which accumulates in its active single chain (SC) form when AEP is absent ${ }^{26,28}$, and other hydrolases such as the active single chain form of CtsD were higher in AEPnull kidney (Fig. 1c).

We investigated this further using kidneys from mice fed a diet where ${ }^{12} \mathrm{C}_{6}$-lysine is replaced by ${ }^{13} \mathrm{C}_{6}$-lysine ('SILAM mice ${ }^{29}$ ) to reveal the proteome of age-matched wildtype (WT) and AEP-null kidney lysosomes (Supplementary Fig. 1a). In addition to proteins we previously detected by $2 \mathrm{D}$-difference gel analysis ${ }^{26}$ including SOD1, transthyretin (Ttr) and Ym1 (Figs. 1c, d, 2 and Supplementary Data 1), mass spectrometric and bioinformatic analysis using the SILAM kidney as a reference sample revealed a striking increase in multiple proteins associated with lysosomes and peroxisomes in the AEP-null kidney (Figs. 1d, 2 and Supplementary Data 1) including cathepsins (Cts) A, B, C, L and $\mathrm{Z}$ and other hydrolases such as hexosaminidase $\mathrm{A}$ and hexosaminidase $\mathrm{B}$, beta mannosidase (Manba) and ganglioside GM2 activator $(\mathrm{Gm} 2 \mathrm{a})$. We validated these changes by direct western blotting of WT and AEP-null kidney lysates (Fig. 1c). Although there was a modest increase in Lamp1 it appeared to be lysosomal contents rather than lysosomal membrane proteins that were increased. Although some differentially represented proteins may be undegraded AEP substrates, for example, fibronectin $(\mathrm{Fn} 1)^{30}$, the general increase in hydrolase levels indicated an unexpectedly coordinated response to deletion of AEP.

This response was not specific to the kidney or to the in vivo state because many of the same proteins such as Gns and CtsD were elevated in SILAC-labelled AEP-null mouse embryonic fibroblasts (MEF; Fig. 1e and Supplementary Fig. 1b). Consistent with these changes, direct protease activity measurements in cell lysates (Fig. 1f) and in intact MEF (Supplementary Fig. 1c) confirmed enhanced $\mathrm{CtsD} / \mathrm{E}$ and $\mathrm{CtsB} / \mathrm{L}$ activity in $\mathrm{AEP}$-null MEF. Moreover, the response could be induced acutely by treatment of WT MEF with the AEP inhibitor MVO26630, (hereafter $\mathrm{MVO}^{31}$ ) which led to increased Gns, CtsD and importantly AEP itself (Fig. 1e, Supplementary Fig. 2a, b and Supplementary Data 1). To assess reversibility of the phenotype, we reconstituted AEP-null MEF with pro-AEP which is taken up and converted to active $\mathrm{AEP}^{32}$. Restoration of AEP activity reversed the accumulations seen in AEP-null MEF (Fig. 1e). Increased protease levels was due to a transcriptional response because chemical AEP inhibition increased mRNA levels of AEP, CtsD and CtsL while genetic deletion of AEP induced even more substantial mRNA increases for CtsD and CtsL (Fig. 1g). Thus, loss of AEP activity promotes its own biosynthesis alongside several other lysosomal hydrolases.

Increased lysosomal proteolytic capacity induced by suppression of AEP activity. These results suggested an interesting and potentially important prediction: AEP ablation might actually lead to faster degradation of substrates traversing the endocytic 

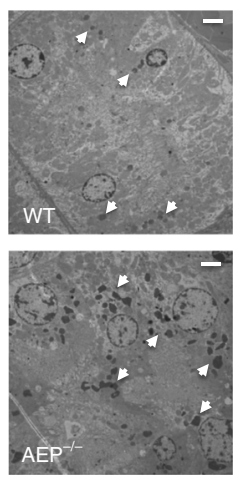

d

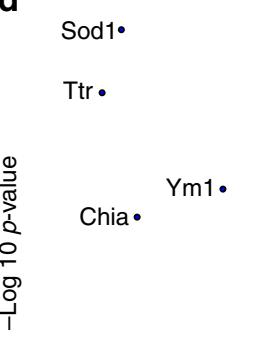

b
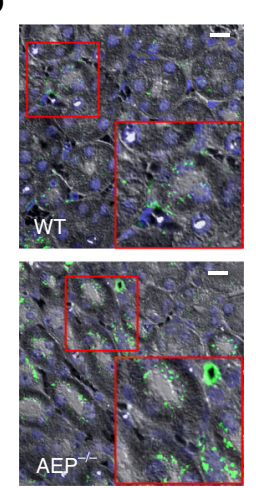

C
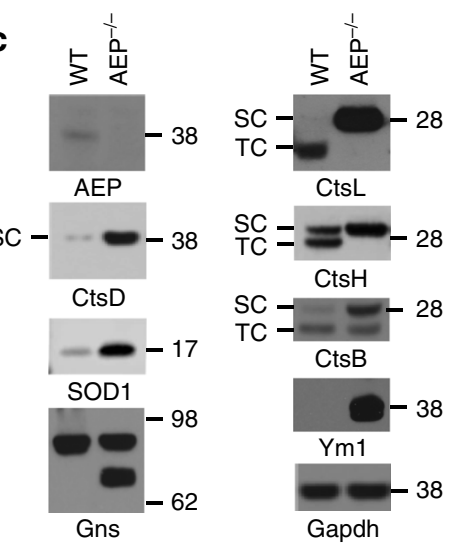

e
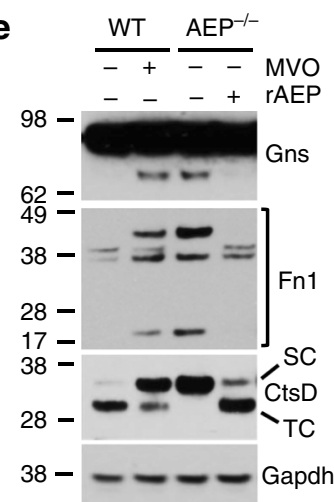

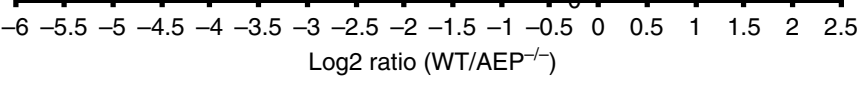
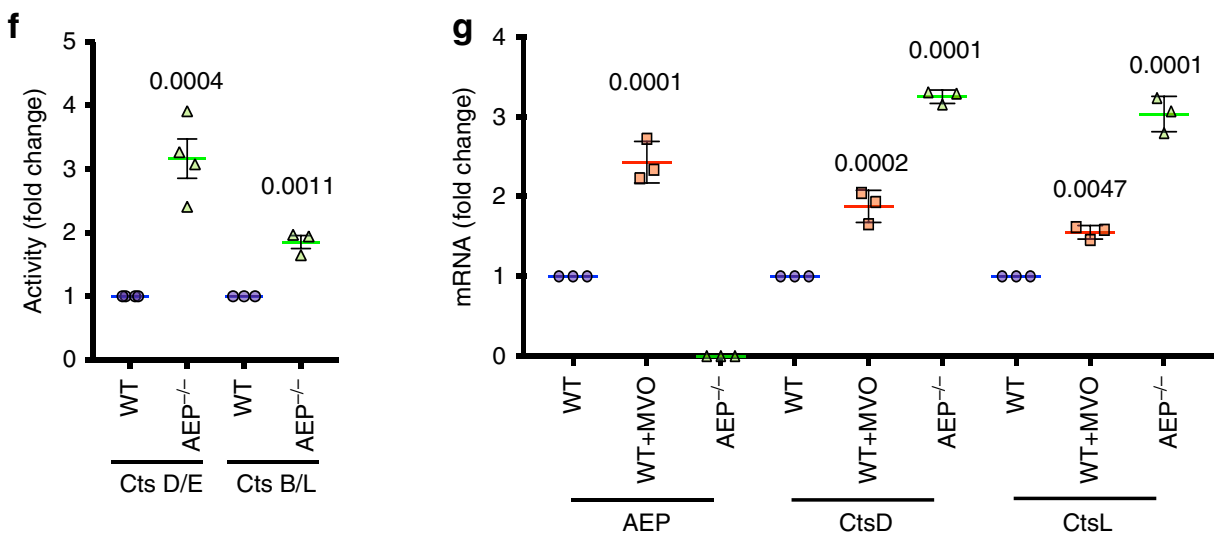

Fig. 1 In vivo SILAC labelling reveals elevated lysosomal hydrolase expression induced by lack of AEP. a Transmission electron microscopy of WT (upper panel) and AEP-/- (lower panel) kidney sections. Arrows indicate lysosomes. (Scale bar $=2 \mu \mathrm{m}$ ) b Immunofluorescence showing more prominent lysosomes (CtsD, green) in AEP $-/$ - kidney sections (lower panel) compared to WT kidney sections (upper panel). (Scale bar $=20 \mu \mathrm{m}$ ). $\mathbf{c}$ Western blot analysis confirming the accumulation in AEP-/- kidneys of proteins identified by mass spectrometry. d Volcano plot showing proteins over-represented (blue dots, lysosomal proteins highlighted in yellow) or under-represented (red dots) in AEP-/- lysosomal kidney fractions against the -log10 $p$-value for three independent experiments. Dotted line on $y$ axis indicates $p$ value $<0.05$. e Acute AEP inhibition with MVO26630 recapitulates hydrolase induction in WT MEF and AEP reconstitution reverses it in AEP-/- MEF. $\mathbf{f}$ Induction of CtsD/E and CtsB/L activities in AEP-/- MEF compared to WT MEF. Data are the average $\pm \mathrm{SD}$ of $n=4$ biologically independent samples for CtsD/E or $n=3$ biologically independent samples for CtsB/L. Statistical significance was calculated using a two-sided unpaired $t$-test. $\mathbf{g}$ Semi-quantitative RT-PCR analysis of mRNA levels for different cathepsins in WT MEF, WT MEF treated overnight with $50 \mu \mathrm{M}$ MVO26630 and AEP-/- MEF. Data are the average \pm SD of $n=3$ biologically independent samples. Statistical significance was calculated using a Dunnett's multiple comparison test

pathway. We tested this for an exogenously supplied substrate and for two endogenous receptors. WT 3T3 fibroblasts, previously incubated with $\mathrm{MVO}$ to block AEP activity, were loaded with DQ-BSA, whose fluorescence is quenched prior to protease action. As shown in Fig. 3a, MVO-treated 3T3s showed stronger fluorescence compared with untreated $3 \mathrm{~T} 3 \mathrm{~s}$ consistent with greater lysosomal protease capacity in the former. Moreover, ligand-induced downregulation of oncostatin $M$ receptor beta $(\mathrm{OSMR} \beta)$ and EGFR was faster in AEP-null and MVO-treated WT MEF compared with WT MEF (Fig. 3b, c). Thus, unexpectedly, genetic or chemical ablation of AEP elicits a response which, overall, leads to increased lysosomal proteolytic capacity. 


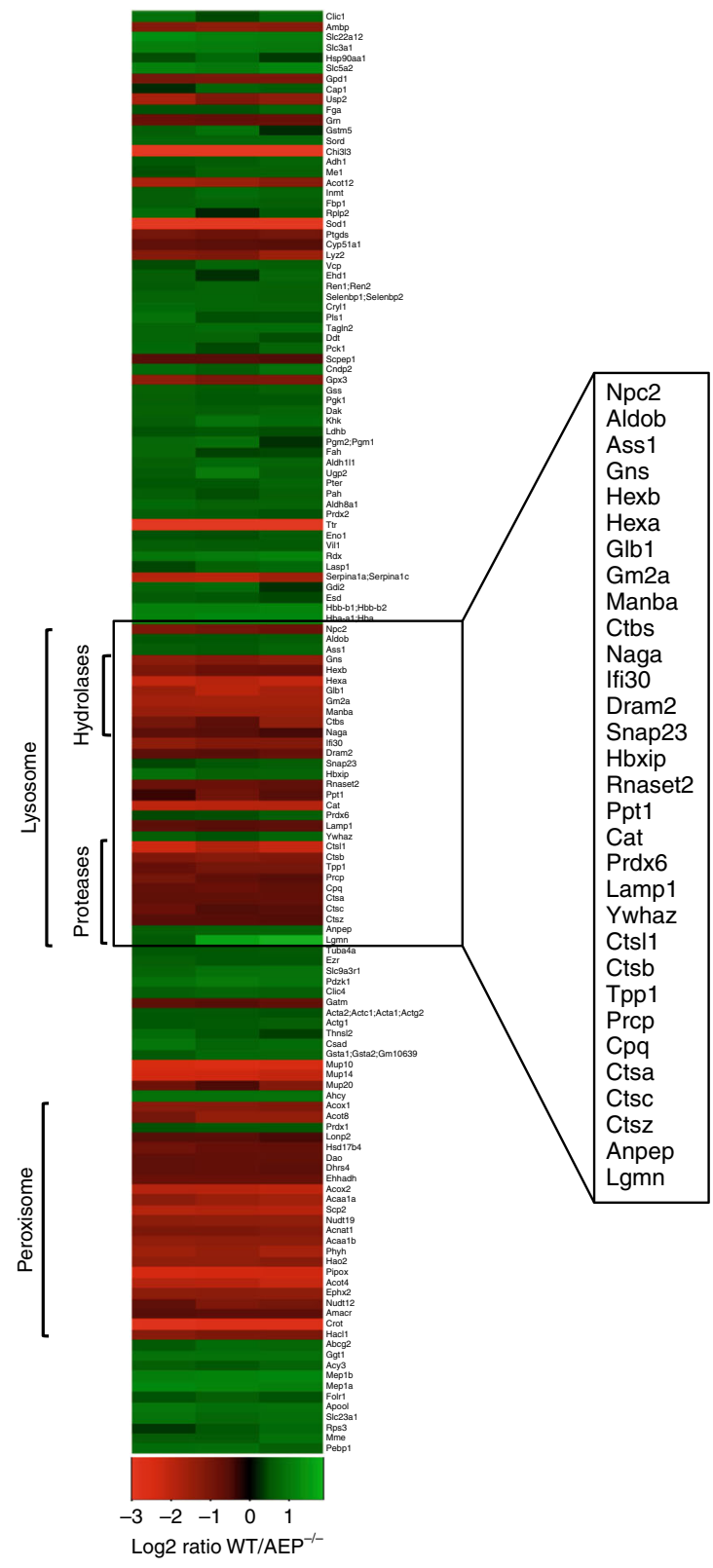

Fig. 2 Lack of AEP induces an increase in the hydrolytic capacity of lysosomes in vivo. Heatmap built using heatmap. 2 in the gplots package of $\mathrm{R}$ program showing proteins over-represented (red) or under-represented (green) in the $\mathrm{AEP}^{-/}$- lysosomal kidney fractions compared to WT samples as identified by our SILAC approach, organised by cellular location and molecular function

AEP suppression triggers a TFEB-independent increase in lysosomal hydrolase expression. We performed various experiments to test whether the upregulation of lysosomal hydrolases following AEP inhibition was due to a TFEB response ${ }^{11,13}$. First, we generated cells expressing TFEB-GFP and challenged them either with MVO or with the mTORC1 inhibitor Torin1. For these and some subsequent experiments, we utilised the human kidney PTC line, HKC- $8^{33}$. As previously reported ${ }^{34}$, TFEB-GFP showed a striking redistribution from cytosol to nucleus upon mTORC1 inhibition (Fig. 4a). In contrast, TFEB remained cytosolic in MVO-treated MEF (Fig. 4a) and in AEP-null kidney PTC (Fig. 4b). In fact, in the latter case TFEB seemed to be excluded from the nucleus to a greater extent compared to WT PTC. This suggested elevated mTORC1 activity and indeed
phospho-4EBP1 levels were higher in AEP-null kidney (Fig. 4c). Reduced mTORC1 activity leads to TFEB activation and downstream induction of autophagy genes ${ }^{13}$. To test further for a TFEB response when AEP was ablated, we examined the autophagy marker LC3 (microtubule-associated protein 1 light chain 3) in MVO, Torin 1 or rapamycin-treated MEF. As expected, inhibition of mTORC1 induced a conversion of LC3-I to the phosphatidyl ethanolamine-conjugated form LC3II consistent with increased autophagic flux. In contrast, LC3 status, and therefore autophagy, was unchanged upon AEP inhibition (Fig. 4d). To investigate this apparent TFEB-independent response further, we used CRISPRCas9 technology to eliminate TFEB expression in HKC-8 cells (Supplementary Fig. 3a). As expected, upon starvation TFEB-null cells no longer induced an increase in cathepsin expression (Supplementary Fig. 3b). In contrast, when the same TFEB-null cells were treated with $\mathrm{MVO}$, there was still a robust increase in expression of CtsD and CtsB at both protein (Fig. 4e) and mRNA (Fig. 4f and Supplementary Fig. 3c) levels. Thus increased lysosomal hydrolytic capacity did not in this instance involve a TFEB response.

Activation of STAT3 in AEP-deficient cells. Further analysis of cytosolic fractions from SILAC-labelled AEP-null MEF revealed a consistent increase both in the level and activation state of the transcription factor STAT3 (Fig. 5a, Supplementary Fig. 4a and Supplementary Data 1). Moreover, inhibition of AEP in WT MEF with MVO or with two other mechanistically distinct AEP inhibitors $^{35}$ induced a dose-dependent increase in activated STAT3 (phospho-Tyr705-STAT3, hereafter P-STAT3) without affecting the levels of total STAT3 (Fig. 5a and Supplementary Fig. 4b). Importantly, P-STAT3 levels were already elevated in AEP-null MEF and were not further increased by MVO demonstrating that STAT3 was activated specifically by suppression of AEP activity (Fig. 5a). Phosphorylation of STAT3 on Tyr705 results in dimerisation and translocation into the nucleus ${ }^{36}$. P-STAT3 was found in the nuclei of AEP-null MEF and MVO-treated WT MEF but not untreated MEF (Fig. 5b). Similarly, in HKC-8 expressing STAT3-YFP, suppression of AEP activity clearly triggered nuclear translocation of STAT3 (Fig. 5b). MVO-treatment of HKC-8 cells also induced a dose-dependent increase in P-STAT3 (Fig. 5c).

STAT3 mediates the lysosomal response to loss of AEP activity. The notion that STAT3 rather than TFEB might control lysosomal hydrolase expression under some conditions seemed plausible since a STAT3-dependent increase in the expression of several cathepsins during the involution of the mammalian mammary gland was recently shown ${ }^{7,8}$. We took multiple approaches to establish a link between STAT3 activation and lysosomal hydrolase expression in our system. Small molecule targeting of STAT3 with S3I-20137 not only blocked STAT3 phosphorylation but almost completely reversed the MVOinduced increase in CtsD and CtsB levels in MEF (Fig. 5d and Supplementary Fig. 5a). To confirm these results, we generated STAT3-depleted HKC-8 cells by siRNA knockdown (Fig. 5e and Supplementary Fig. 5b). Knockdown of STAT3 by two different siRNAs suppressed the MVO-induced increase in CtsB and CtsD protein levels, a result not seen with a non-targeting siRNA control (Fig. 5e and Supplementary Fig. 5b). Further, we also generated a STAT3 mutant (fused to GFP to distinguish it from endogenous STAT3) designed to resist siRNA knockdown (mSTAT3-GFP). MVO-induced induction of CtsD was rescued in cells expressing mSTAT3-GFP, which in contrast to endogenous STAT3, was largely resistant to siRNA knockdown (Supplementary Fig. 5c) demonstrating unequivocally a requirement for STAT3. Consistent with canonical activation of STAT3, 
a
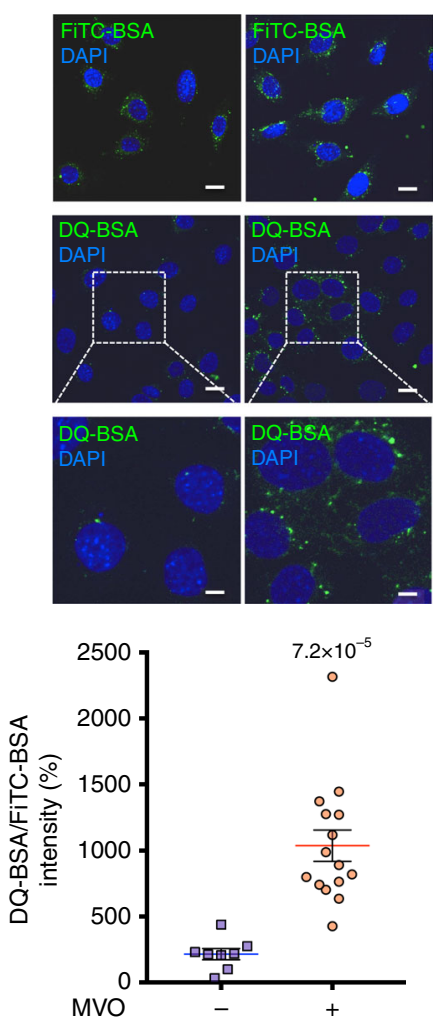

b
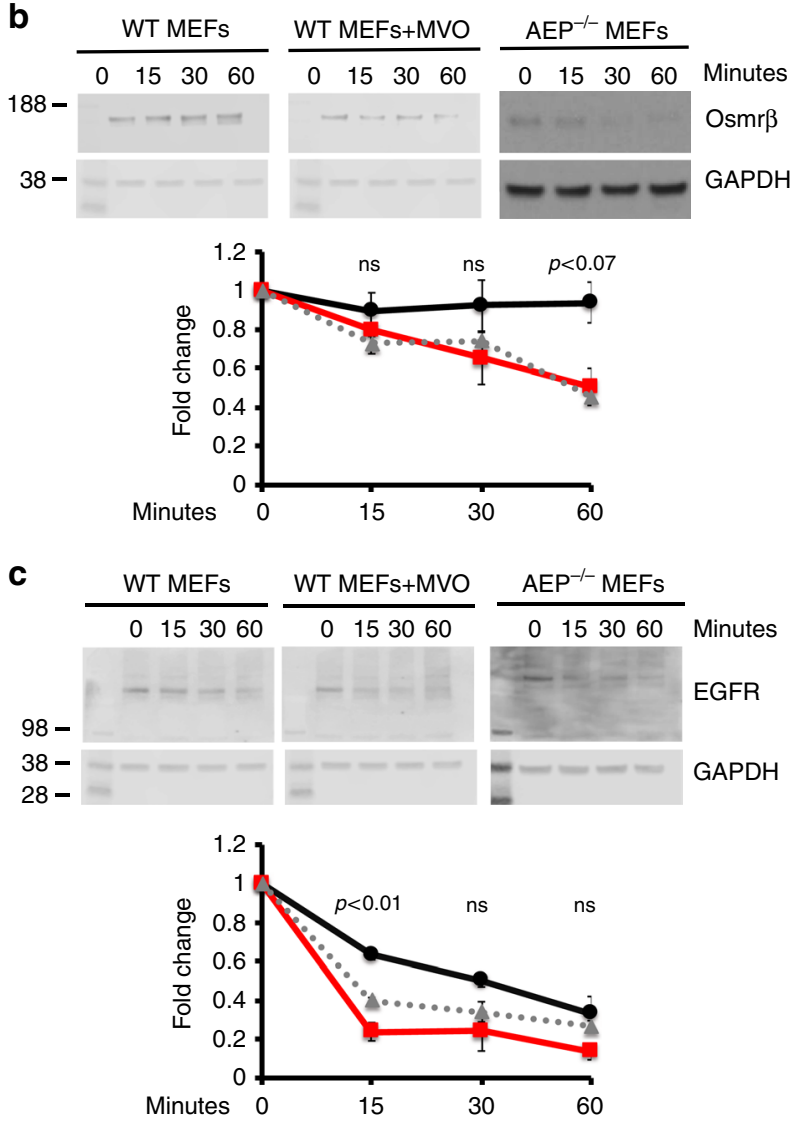

Fig. 3 Increased lysosomal proteolytic capacity induced by suppression of AEP activity. a FiTC-BSA uptake and DQ-BSA degradation rates in WT 3T3s

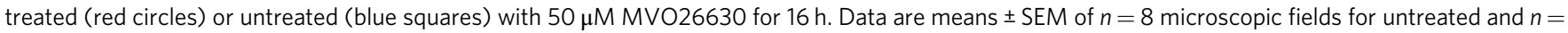
15 microscopic fields for MVO-treated. (Scale bar $=20 \mu \mathrm{m})$. b Time course of degradation of oncostatin M receptor beta (Osmr $\beta$ ) in WT MEFs treated (dashed grey line) or untreated (black solid line) with $50 \mu \mathrm{M} \mathrm{MVO26630}$ for $16 \mathrm{~h}$ and AEP ${ }^{-/-}$MEFs (red solid line) following receptor downregulation by oncostatin M. Relative intensity of each Osmr $\beta$ band was calculated for three independent experiments. c Time course of degradation of epithelial growth factor receptor (EGFR) in WT MEFs treated (dashed grey line) or untreated (black solid line) with $50 \mu \mathrm{M} \mathrm{MVO} 26630$ for $16 \mathrm{~h}$ and AEP-/- MEFs (red solid line) following receptor downregulation by epithelial growth factor. Relative intensity of each EGFR band was calculated for three independent experiments. Statistical significance was calculated using a two-sided unpaired $t$-test. ns $=$ not significant

inhibition of Jak2 with the inhibitor SD-1029 suppressed MVOinduced STAT3 phosphorylation (Fig. $5 \mathrm{f}$ and Supplementary Fig. 5d). Moreover, MVO treatement of HKC-8 cells activated Jak2, as indicated by enhanced phosphorylation on Tyr1007/1008 (Fig. 5g). To confirm the involvement of Jak2, we generated Jak2depleted cells. As shown in Fig. 5h and Supplementary Fig. 5e, two different siRNAs directed to Jak2, but not a non-targeting siRNA, suppressed MVO-induced STAT3 phosphorylation as well as increased levels of CtsB and CtsD protein (Fig. 5h and Supplementary Fig. 5e). Thus, ablation of AEP activates the canonical Jak2-STAT3 pathway and leads to upregulation of other lysosomal hydrolases.

Direct promotion of lysosomal hydrolase expression by STAT3. Overall, $67 \%$ of proteins increased 1.5 -fold or more in AEP-null MEFs featured a canonical STAT3 site in their promoters while almost $90 \%$ contained either canonical or noncanonical STAT3 sites as defined in a recent study ${ }^{38}$. Although this was consistent with STAT3 recruitment to lysosomal hydrolase promoters it was important to test this directly in AEP $-l-$ versus WT cells. We immunoprecipitated STAT3-associated chromatin fragments (ChIP) from WT and $\mathrm{AEP}^{-/-} \mathrm{MEF}$ and compared the signal obtained by PCR from input versus precipitated DNA for key upregulated hydrolase genes. We observed enhanced STAT3 recruitment to the promoters of CtsD, CtsL, the still intact promoter region of the AEP gene and other hydrolase genes in $\mathrm{AEP}^{-1-}$ compared with WT cells (Fig. 6a, b and Supplementary Fig. 6a). STAT3 recruitment to the AEP promoter made sense of the initially puzzling observation that low levels of MVO increased, rather than decreased AEP activity in cells. Blockade of STAT3 with S3I-201 prevented this increase and increased MVO potency at all concentrations (Fig. 6c). Taken together, these results demonstrate direct involvement of STAT3 in regulation of lysosomal hydrolase expression when a key lysosomal hydrolase activity is missing. Importantly, elevated activity of AEP and CtsD was also observed in 3T3 fibroblasts (Fig. 6f) expressing a constitutively active form of STAT3, STAT3c, which dimerises in the absence of tyrosine phosphorylation 39,40 , an increase which was again due to increased protease mRNA levels in these cells (Fig. 6d and Supplementary Fig. 6b).

Protein overload induces STAT3-dependent protease expression. If the response triggered by AEP ablation is due to accumulation of undegraded substrates, we reasoned that a similar response should be induced by substrate overload of the endocytic pathway. To test this WT MEFs were incubated for $16 \mathrm{~h}$ with $30 \mathrm{mg} / \mathrm{ml} \mathrm{BSA}$ and the status of STAT3 as well as protease 
a

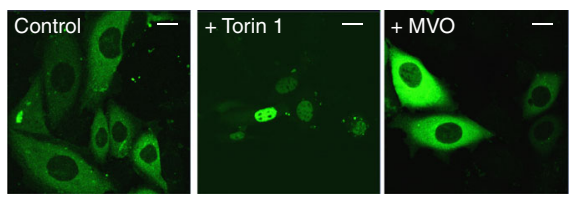

b
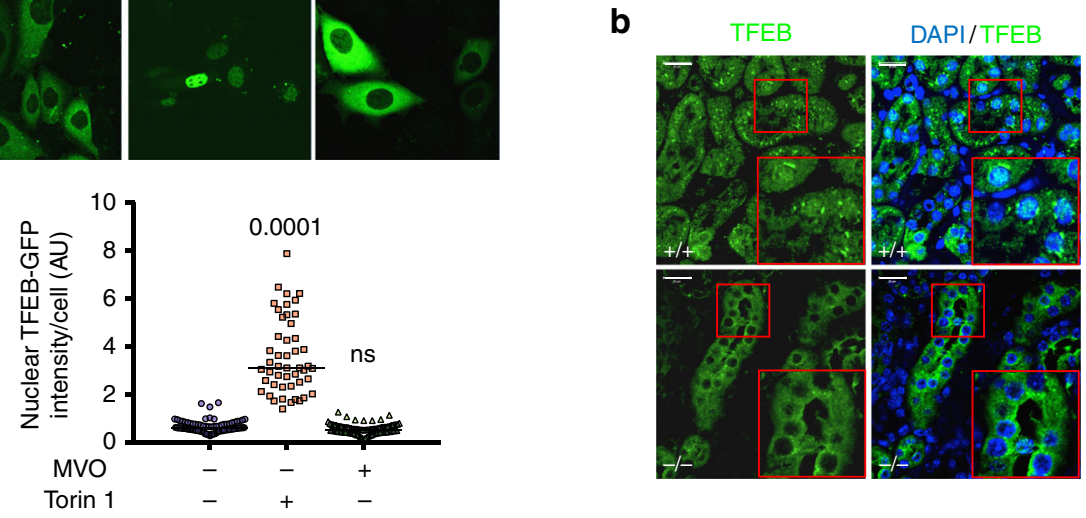

d
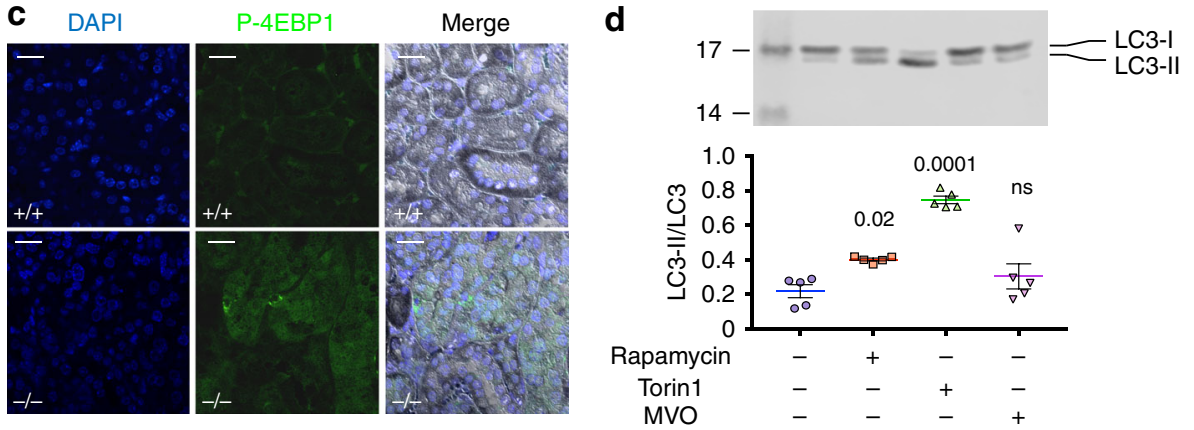

e
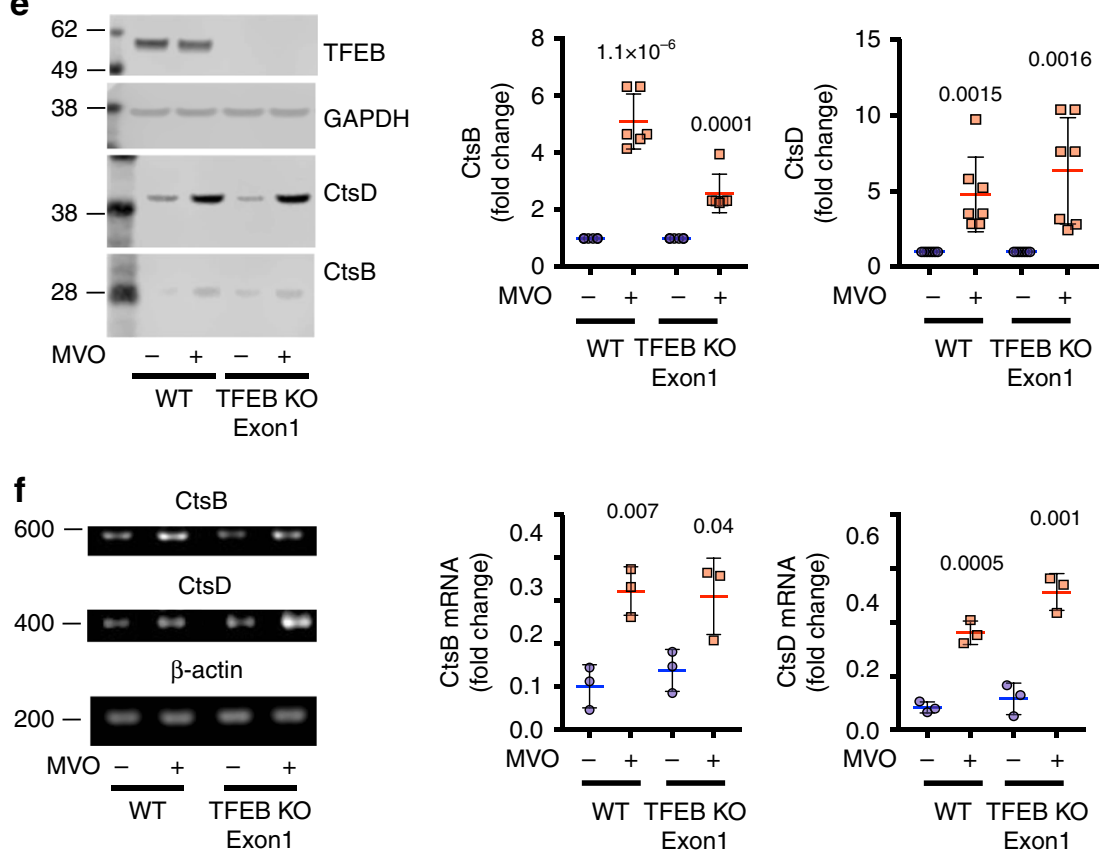

Fig. 4 Increased lysosomal hydrolytic capacity induced by chronic or acute AEP-deficiency does not depend on TFEB activation. a Cellular localisation of TFEB-GFP in HKC-8 cells treated or untreated with $50 \mu \mathrm{M}$ MVO26630 or $1 \mu \mathrm{M}$ Torin1 overnight. (Scale bar $=20 \mu \mathrm{m}$ ). Data are the average \pm SD of $n=93$ cells for control sample, $n=48$ for Torin1-treated sample and $n=92$ for MVO-treated sample. Statistical significance was calculated using a Dunnett's multiple comparison test. b Localisation of TFEB by IF in WT and AEP-/- kidney sections. (Scale bar $=20 \mu \mathrm{m}$ ). c Levels of P-4EBP1 by IF in WT and $\mathrm{AEP}^{-1-}$ kidney sections. (Scale bar $=20 \mu \mathrm{m}$ ). $\mathbf{d}$ Immunoblotting and quantitation of LC3II/total LC3 ratio in lysates from WT MEFs treated with Rapamycin $(1 \mu \mathrm{M})$ or Torin1 $(1 \mu \mathrm{M})$ for $4 \mathrm{~h}$ or with MVO26630 $(50 \mu \mathrm{M})$ for $16 \mathrm{~h}$. Data are the average \pm SD of $n=5$ biologically independent samples. Statistical significance was calculated using a Dunnett's multiple comparison test. e Levels of expression of CtsB and CtsD in HKC-8 WT and TFEB

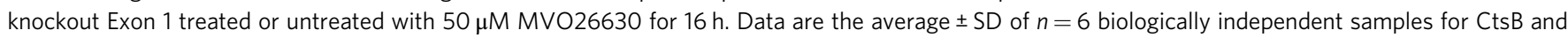
$n=7$ biologically independent samples for CtsD. Statistical significance was calculated using a two-sided unpaired $t$-test. $\mathbf{f}$ mRNA expression levels for CtsB and CtsD in HKC-8 WT and TFEB knockout Exon 1 treated or untreated with $50 \mu$ M MVO26630 for 16 h. Data are the average \pm SD of $n=3$ biologically independent samples. Full data and gel calibration markers can be seen in Supplementary Fig. 3c. Statistical significance was calculated using a two-sided unpaired $t$-test 
a

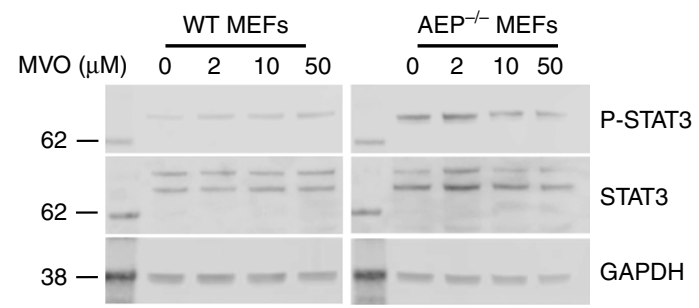

d

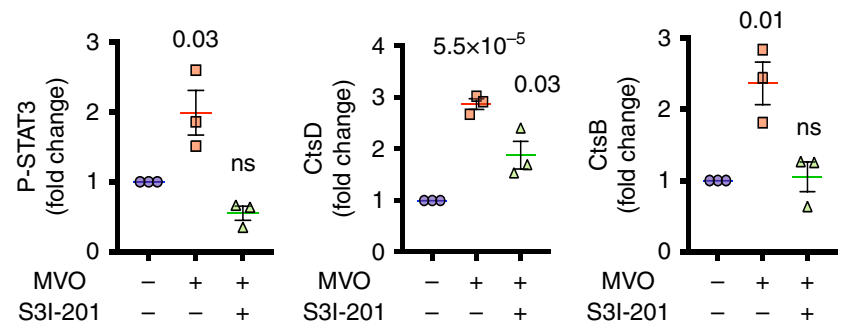

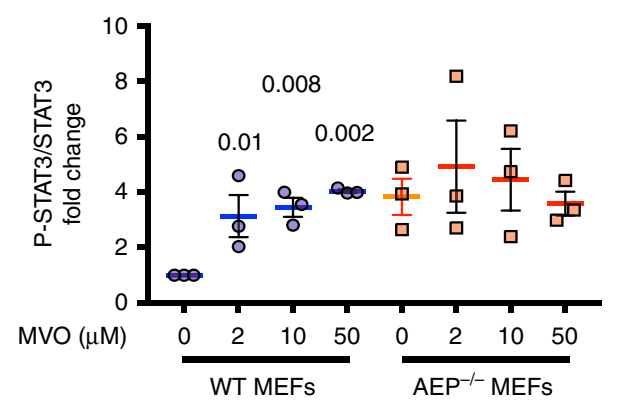
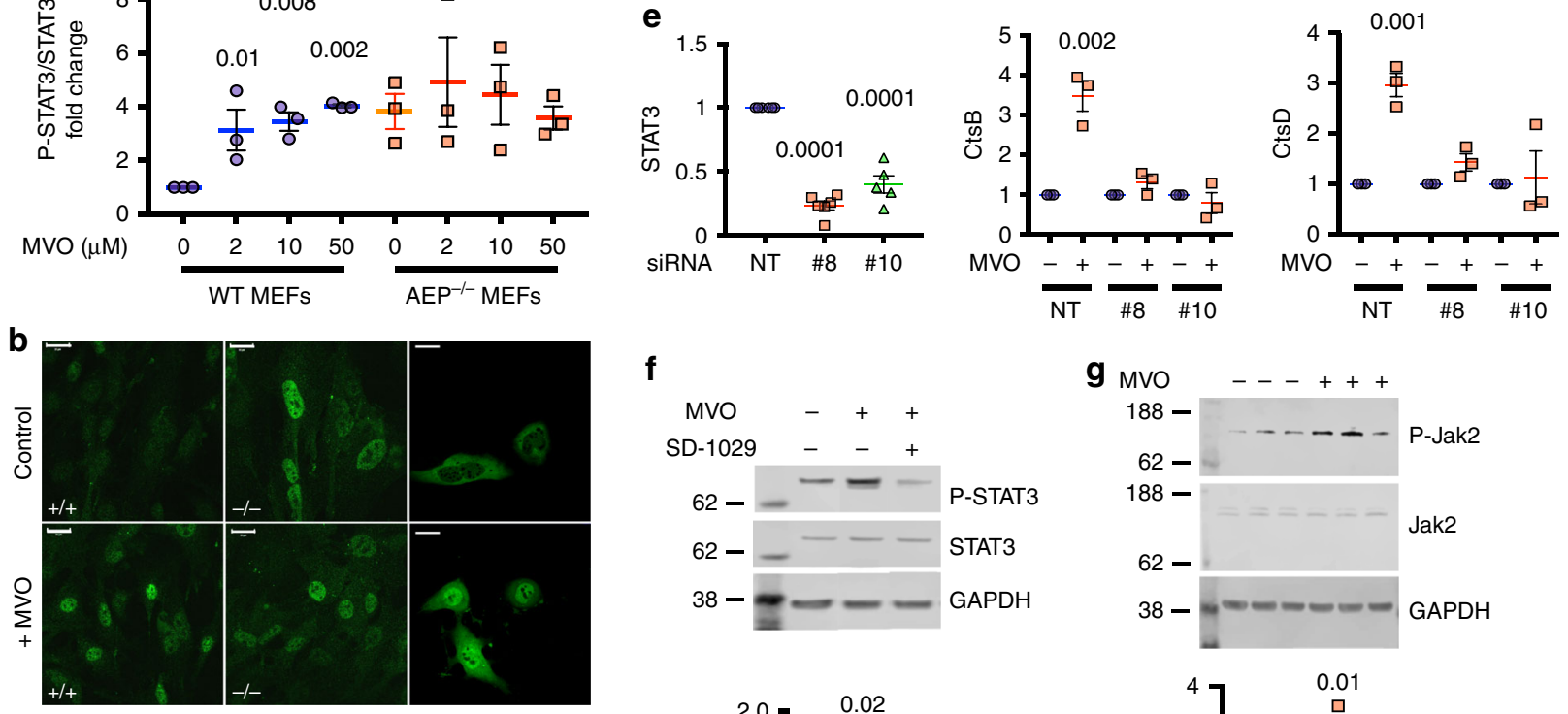

f
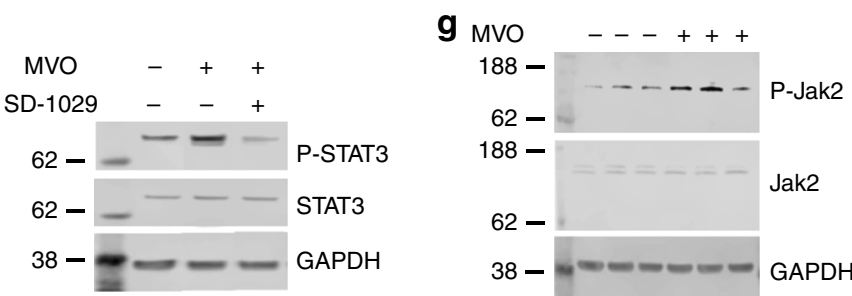

Endogenous P-STAT3

STAT3-YFP
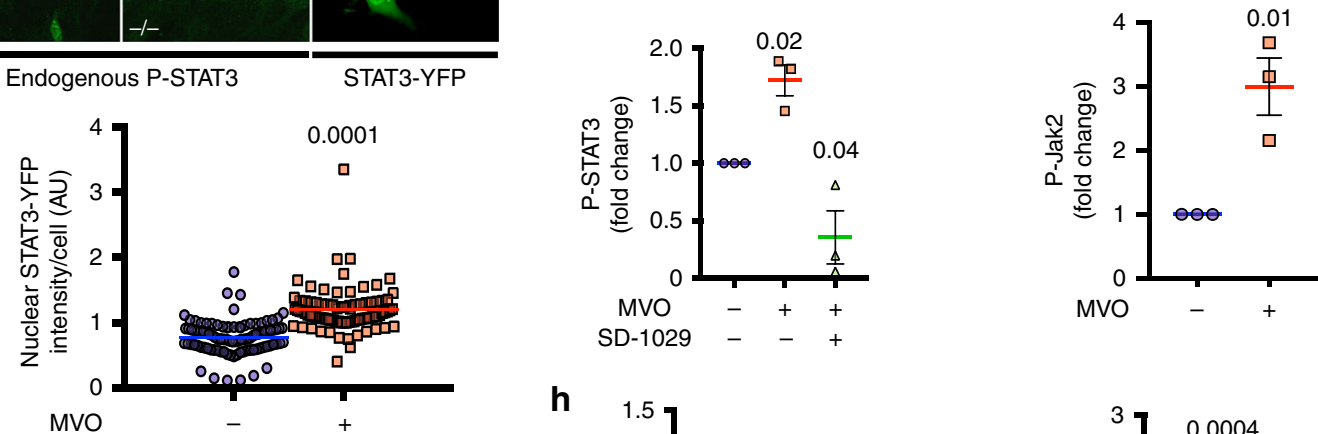

C
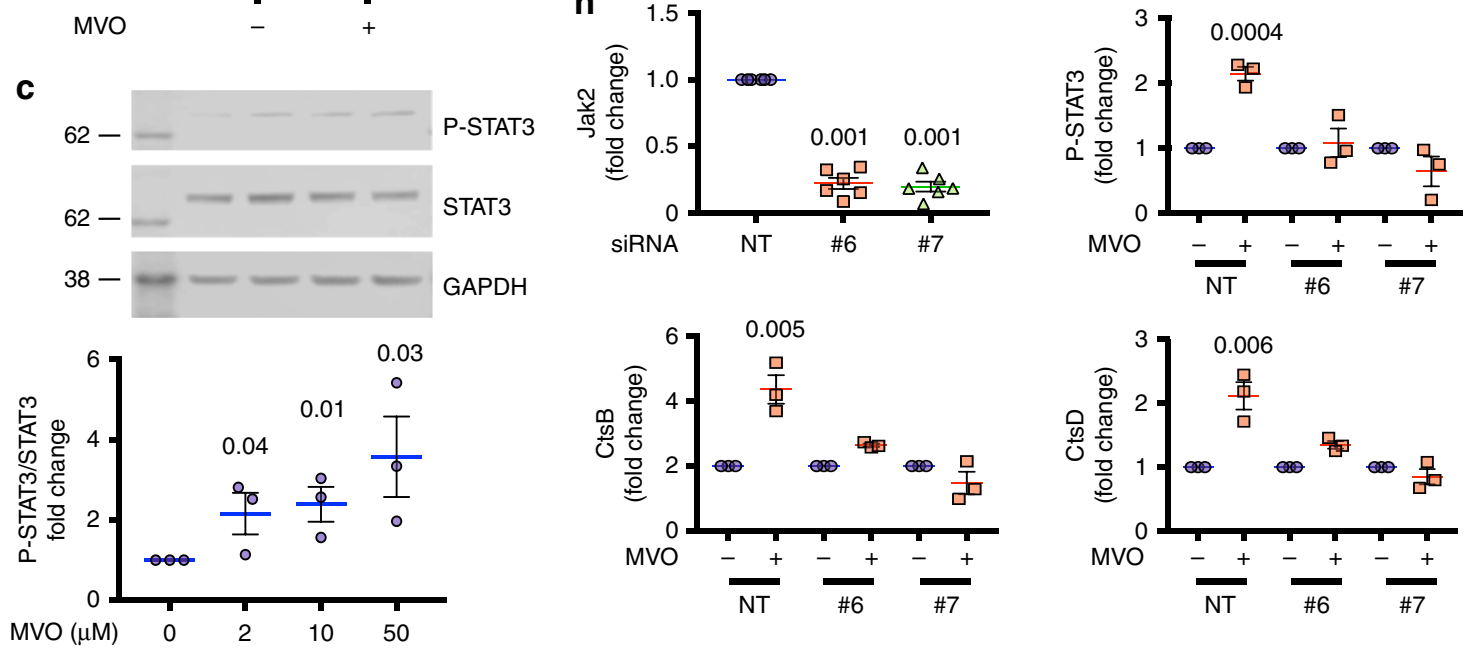

mRNA levels and activities assessed. Protein overload led to a two to sevenfold increase in the mRNA levels of several lysosomal proteases including AEP, CtsB, CtsL, CtsD and CtsH (Fig. 6e and Supplementary Fig. 7) consistent with an increase in protease activity levels (Fig. 6g). This increase was blunted by STAT3 inhibition with S3I-201 (Fig. 6g). Importantly, increased protein load also led to a striking increase in nuclear P-STAT3 similar to that seen upon MVO-treatment (Fig. 6h) but without nuclear translocation of TFEB (Supplementary Fig. 8a). In contrast, mTORC1 inhibition with either rapamycin or Torin1 had no 
Fig. 5 Increased lysosomal hydrolytic capacity induced by chronic or acute AEP-deficiency is controlled by the Jak2-STAT3 signalling pathway. a STAT3 activation in WT but not AEP-/- MEFs treated for $16 \mathrm{~h}$ with increasing concentrations of MVO26630. Data are the average \pm SD of $n=3$ biologically independent samples. Statistical significance was calculated using a Dunnett's multiple comparison test. b P-STAT3 localisation in WT and AEP-/- MEFs treated or not with $50 \mu \mathrm{M}$ MVO26630 for $16 \mathrm{~h}$ (endogenous P-STAT3) and HKC-8 cells transfected with STAT3-YFP and treated or not with $50 \mu \mathrm{M}$ MVO26630 for $16 \mathrm{~h}$. Data are the average \pm SEM of $n=96$ cells for control samples and $n=86$ for MVO-treated samples. Statistical significance was calculated using a two-sided unpaired $t$-test. (Scale bar $=20 \mu \mathrm{m}$ ). c STAT3 activation in HKC-8 cells treated with increasing levels of MVO26630 overnight. Data are the average \pm SD of $n=3$ biologically independent samples. Statistical significance was calculated using a two-sided unpaired $t$-test. d CtsD, CtsB and P-STAT3 levels in WT MEFs compared to WT MEFs treated overnight with $50 \mu \mathrm{M} \mathrm{MVO26630}$ with or without $100 \mu \mathrm{M}$ of the STAT3 inhibitor S3I-201. Data are the average \pm SD of $n=3$ biologically independent samples. Statistical significance was calculated using a two-sided unpaired $t$ test. e Levels of STAT3, CtsB and CtsD in WT HKC-8 cells transfected with a non-targeting siRNA (NT) or two different STAT3-targeting siRNAs (\#8 and \#10) and treated or not with $50 \mu \mathrm{M}$ MVO26630 for $16 \mathrm{~h}$. Data are the average \pm SD of $n \geq 3$. Statistical significance was calculated using a two-sided unpaired $t$-test. f P-STAT3 western blot in WT HKC-8 treated with $50 \mu \mathrm{M}$ MVO26630 in the presence or absence of the Jak2 inhibitor SD-1029 (10 $\mu$ M). Data are the average \pm SD of $n=3$ biologically independent samples. Statistical significance was calculated using a Dunnett's multiple comparison test. g Levels of P-Jak2 in WT HKC-8 cells treated or untreated with $50 \mu \mathrm{M}$ MVO26630 for $16 \mathrm{~h}$. Data are the average \pm SD of $n=3$ biologically independent samples. Statistical significance was calculated using a two-sided unpaired $t$-test. $\mathbf{h}$ Levels of Jak2, P-STAT3, CtsB and CtsD in WT HKC-8 cells transfected with a non-targeting siRNA (NT) or two different Jak2-targeting siRNAs (\#6 and \#7) and treated or not with $50 \mu \mathrm{M}$ MVO26630 for $16 \mathrm{~h}$. Data are the average \pm SD of $n \geq 3$ biologically independent samples. Statistical significance was calculated using a two-sided unpaired $t$-test

effect on P-STAT3 distribution but instead triggered focalised LC3 accumulation on autophagosomes, a response that was not observed either with MVO-treatment or protein overload (Fig. 6h). This important result demonstrates that STAT3-driven expression of lysosomal hydrolases is not a limited response to the perturbations caused by loss of AEP activity but one induced by increased load on the lysosomal system and therefore likely to be of widespread physiological significance. Moreover, the protease/substrate imbalance conditions that activate STAT3 do not signal through the mTORC1/TFEB pathway and conversely, suppression of mTORC1 activity which activates TFEB does not activate STAT3 (Fig. 6h).

Lysosomal stress triggers STAT3-dependent de novo protease expression. STAT3 can be activated by a variety of exogenous and endogenous stimuli including oxidative stress ${ }^{41,42}$. We postulated that oxidative stress following acute AEP inhibition might be a driver of STAT3 activation since oxidative stress is a common feature of lysosomal storage diseases ${ }^{43}$. Consistent with this scenario, SOD1 levels were elevated in both AEP-null kidney ${ }^{26}$ and MEF (Fig. 1c, d and Fig. 7a), and SOD1 activity was also boosted following acute AEP inhibition with MVO (Fig. 7b). We strengthened the link between AEP-deficiency, oxidative stress and STAT3 activation in three ways. First, the 'spin trap' antioxidant phenyl- $\alpha$-tert butyl nitrone (PBN) completely blocked the activation of STAT3 (Fig. 7c). Second, direct exposure to $\mathrm{H}_{2} \mathrm{O}_{2}$ induced the nuclear translocation of STAT3-YFP but not TFEBGFP in HKC-8 cells (Fig. 7d) and moreover, increased the level of CtsD (Fig. 7e). Indeed, in $\mathrm{H}_{2} \mathrm{O}_{2}$ treated MEF, increased mRNA levels for multiple lysosomal proteases were observed (Fig. $7 \mathrm{f}$ and Supplementary Fig. 7). Third, CellROX Orange, a reporter dye that fluoresces upon oxidation showed a twofold increase in lysosomal labelling in AEP-null MEF and in WT MEF treated with MVO compared to untreated WT MEFs (Fig. 7g), thus identifying lysosomes as sites of ROS generation. Importantly, protein overload of lysosomes also induced oxidative stress signals in lysosomes (Supplementary Fig. 8b), underlining that STAT3 activation by lysosomal stress is a general response aimed at recovering lysosomal homoeostasis induced by lysosomal protease/substrate imbalance.

AEP ablation is linked to STAT3 activation in murine kidney PTC in vivo and in human PTC. To test the physiological relevance of the STAT3-driven response to lysosomal stress, we re-examined kidneys from AEP-null mice and mice expressing constitutively activated STAT3c. Indeed, levels of P-STAT3 were higher in AEP-null kidney compared with WT although levels of STAT3 protein appeared similar (Fig. 8a). Importantly, labelling for P-STAT3 was particularly prominent in the PTC, which exhibits the highest levels of AEP expression ${ }^{26}$ (Fig. 8b). This finding most likely explains the hyperproliferative PTC phenotype observed in AEP-null kidney ${ }^{26}$ and confirmed here using anti-Ki67 staining (Fig. 8c). Once again, increased expression of lysosomal hydrolases could be uncoupled from AEP status since kidneys from STAT3c mice ${ }^{44}$ also showed striking increases in many of the proteins that were induced by AEP-deficiency or protein overload including CtsD and CtsL, Ym1, Gns and AEP (Fig. 8d). To test whether the response was conserved in primary human kidney cells, we expanded PTC as well as distal tubular cells (DTC) from biopsied human kidneys. MVO-treatment induced STAT3 activation and increased expression of CtsD in primary PTC but not in DTC (Fig. 8e).

STAT3 regulation of lysosomal capacity in cathepsin depleted cells and tissues. Finally, we addressed two further important questions: does ablation of lysosomal proteases besides AEP induce STAT3-driven hydrolase expression and do tissues other than the kidney show evidence of this response? First, we looked for an acute in vitro response akin to that induced by MVO by treating WT MEF with the cysteine cathepsin inhibitor E64 or the aspartyl cathepsin inhibitor pepstatin. WT MEF treatment with pepstatin increased P-STAT3 levels and boosted CtsD expression substantially as did E64 albeit to a lesser extent (Fig. 9a). Next, we examined tissues from mice genetically ablated for CtsL or B which are both E64 targets. We examined heart since previous studies had demonstrated a cardiomyopathic phenotype in ageing CtsL-null mice and enlarged lysosomes in the myocardium ${ }^{45}$. In CtsL-null heart (Fig. 9b), P-STAT3 and CtsD levels were increased by at least a factor of 2 (Fig. 9b). Similar increases were observed in CtsB-null heart tissue although no overt heart phenotype has been described in these mice. Moreover, as in cells and tissues lacking AEP, SOD1 expression was also increased in Ctsnull tissues providing evidence that oxidative stress was a factor once again (Fig. 9b). Thus, ablation of diverse lysosomal protease activities can induce a STAT3-regulated response whose purpose is presumably to restore homoeostasis.

\section{Discussion}

How cells tune the performance of their lysosomes to meet the demand placed on them has only recently been investigated. A particular challenge is to understand how the environment in the lysosomal lumen is sensed and communicated to cytosolic 
a

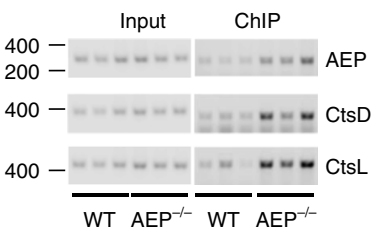

b 20

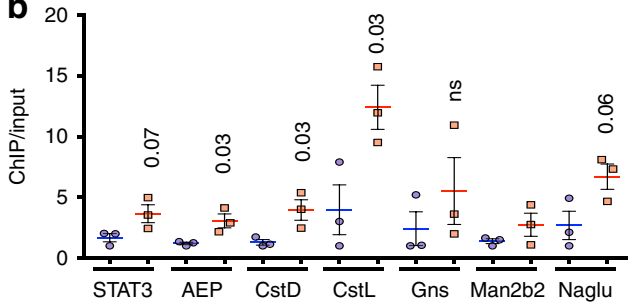

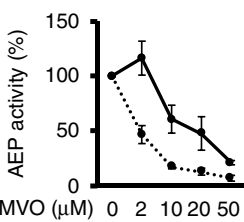
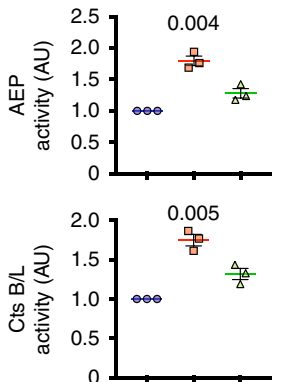
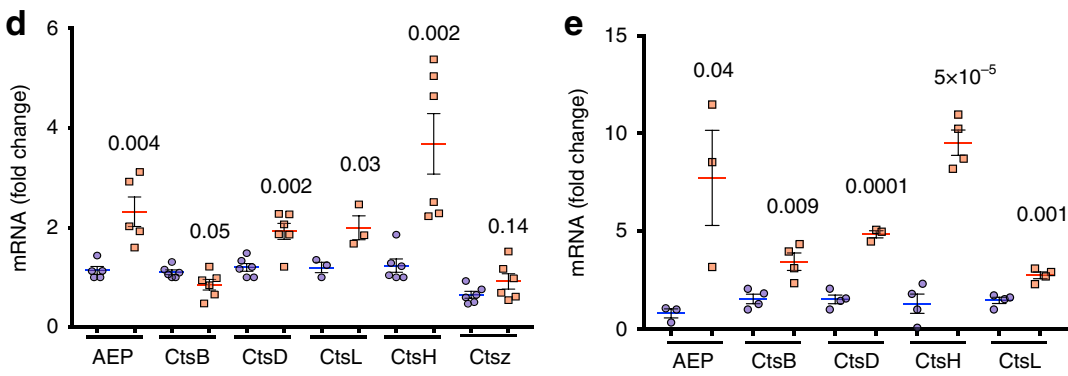

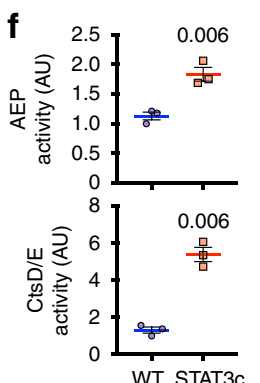

g

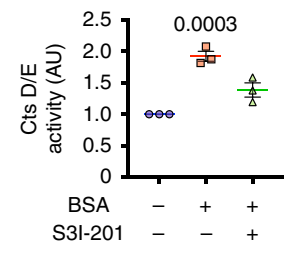

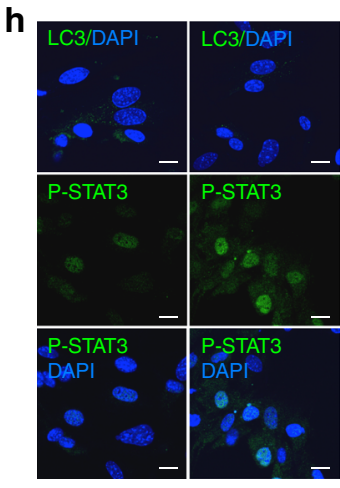

Control

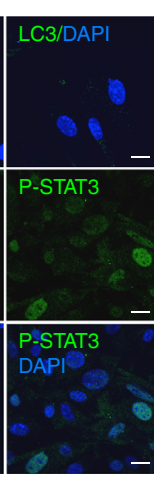

$+\mathrm{MVO}$

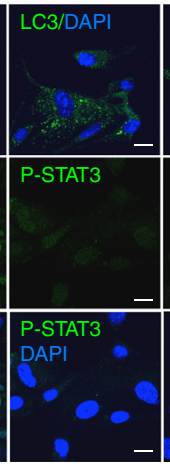

+ Rapamycin

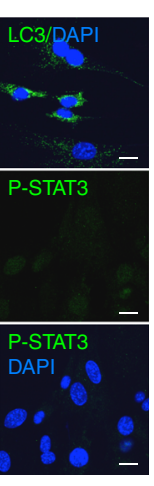

+ Torin 1
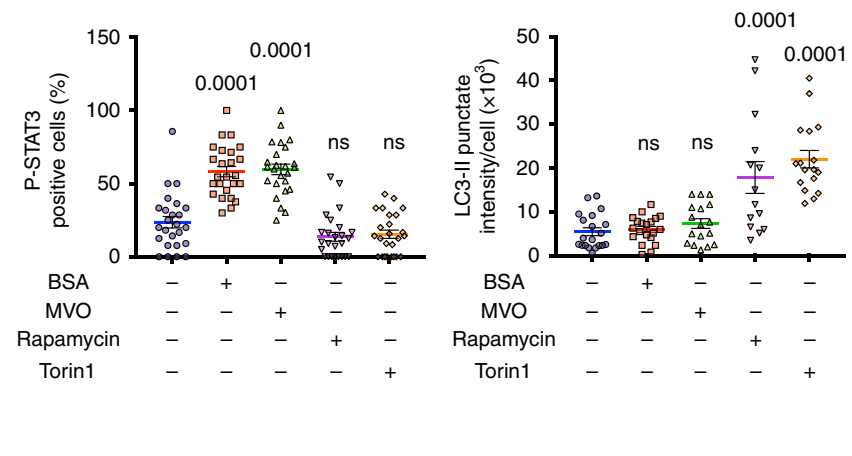

Fig. 6 Direct STAT3 involvement in regulation of lysosomal hydrolase genes under conditions of protease activity/lysosomal substrate imbalance. a Agarose gel analysis of representative ChIP-PCR products for AEP, CtsD and CtsL promoters in WT and AEP-deficient MEFs. Full data and gel calibration markers can be seen in Supplementary Fig. 6. b Quantitative PCR analysis of DNA from STAT3 ChIP of WT (blue circles) and AEP-deficient (red squares) MEFs expressed as fold enrichment. Data are the average \pm SD of $n=3$ biologically independent samples. Statistical significance was calculated using a two-sided unpaired t-test. c AEP activity in WT MEF treated overnight with different concentrations of MVO26630 in the presence (dashed line) or absence (solid line) of $100 \mu \mathrm{M} \mathrm{S3I-201.} \mathrm{Data} \mathrm{are} \mathrm{the} \mathrm{average} \pm$ SD of three independent experiments. d Quantitative PCR analysis of the mRNA levels for different lysosomal proteases in WT (blue circles) and STAT3c 3T3 (red squares) cells. Data are the average \pm SD of $n=6$ biologically independent samples for all samples, except for AEP where $n=5$ and CtsL where $n=3$. Statistical significance was calculated using a two-sided unpaired $t$-test. e Quantitative PCR analysis of the mRNA levels for different lysosomal proteases in WT MEFs treated (red squares) or not (blue circles) with 30 mg/ml BSA overnight. Data are the average \pm SD of $n=4$ biologically independent samples for all samples, except for AEP and CtsD where $n=3$. Statistical significance was calculated using a two-sided unpaired t-test. $\mathbf{f}$ AEP and CtsD/E activities measured in WT and STAT3c 3T3 cells. Data are the average $\pm \mathrm{SD}$ of $n=3$ biologically independent samples. Statistical significance was calculated using a two-sided unpaired $t$-test. $\mathbf{g}$ Activities of AEP, CtsB/L and CtsD/E in WT MEF treated or not with $30 \mathrm{mg} / \mathrm{ml} \mathrm{BSA}$ overnight in the presence or absence of $100 \mu \mathrm{M} \mathrm{S3I-201}$. Data are the average \pm SD of $n=3$ biologically independent samples. $\mathbf{h}$ Quantitation of P-STAT3 and LC3II levels in WT MEFs treated with Rapamycin (1 $\mu$ M) or Torin1 (1 $\mu$ M) for $4 \mathrm{~h}$ or with MVO26630 $(50 \mu \mathrm{M})$ or BSA $(30 \mathrm{mg} / \mathrm{ml})$ for $16 \mathrm{~h}$. Data are the average \pm SD of $n=25$ microscopic fields for P-STAT3 and $n=14$ for LC3II. Statistical significance was calculated using a Dunnett's multiple comparison test. ns $=$ not significant. (Scale bar $=20 \mu \mathrm{m})$

signalling pathways and the transcriptional machinery. Our results show that when the capacity of the lysosome to 'keep up' with substrate load is compromised, either because of loss of a key hydrolytic activity or because of increased endocytic load, oxidative stress leads to activation of STAT3 and the de novo transcription of a variety of lysosomal hydrolases. Although the endpoint is similar, this pathway differs fundamentally from the recently elucidated mTORC1-TFEB pathway for lysosomal biogenesis ${ }^{46}$.

A mechanism for enhancing lysosomal capacity independent of the mTORC1-TFEB pathway makes sense from several standpoints. In particular, the cellular response to nutrient poor conditions that promote autophagy would be expected to be different to the response to conditions which can be characterised as 'nutrient rich' where exogenous proteins are abundant. Recent studies have shown that cells can use bulk endocytic uptake pathways such as macropinocytosis to capture proteins and degrade them to meet nutritional demands and to drive cell proliferation ${ }^{47,48}$. Under these conditions, enhanced generation of amino acids in the lysosomal lumen would be expected to maintain or even enhance mTORC1 activity precluding TFEB activation. Indeed, most likely as a result of enhanced lysosomal protein breakdown and amino acid availability, we observed tight retention of TFEB on lysosomes under conditions that induced STAT3 activation.

Although 'nutrient poor' and 'protein nutrient rich' conditions both benefit from enhanced lysosomal capacity, other aspects of cellular physiology in these states are quite different. For example, 

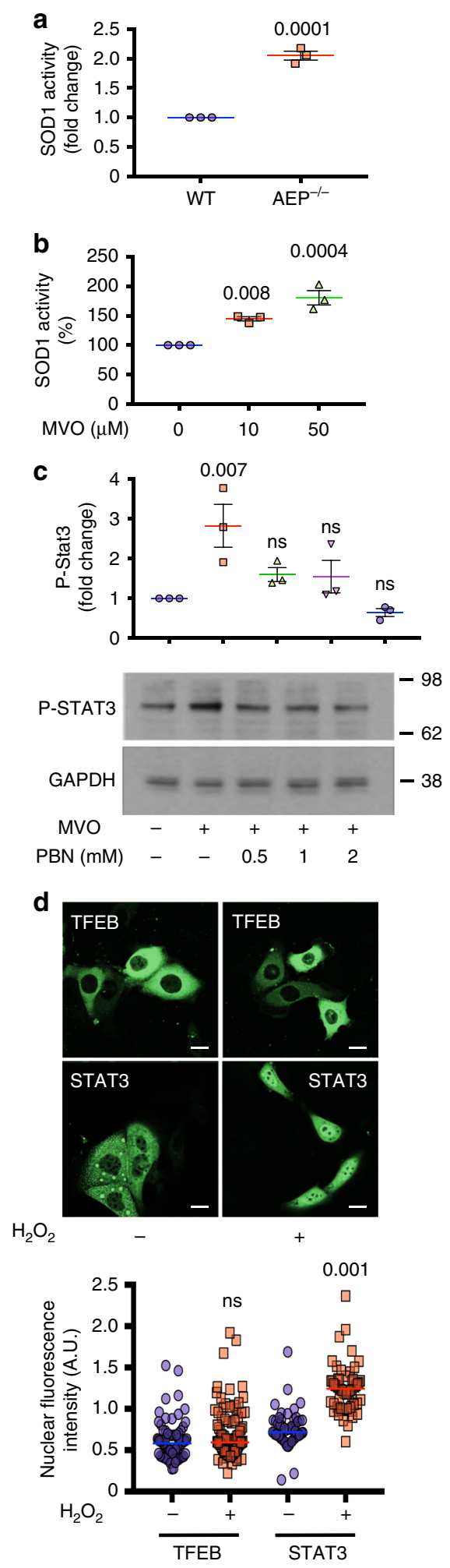

a number of TFEB target genes are involved in autophagy ${ }^{13}$. Induction of autophagy alongside increased lysosomal capacity makes sense under nutrient poor conditions but probably not under conditions of increased substrate load since this would likely overload and stress the lysosomal system still further. In fact, faced with lysosomal overload of undegraded substrates, it would make sense to temporarily inhibit constitutive autophagy which is ongoing in most cells ${ }^{49}$. Consistent with this, there is extensive evidence that autophagy is indeed inhibited by STAT3, e

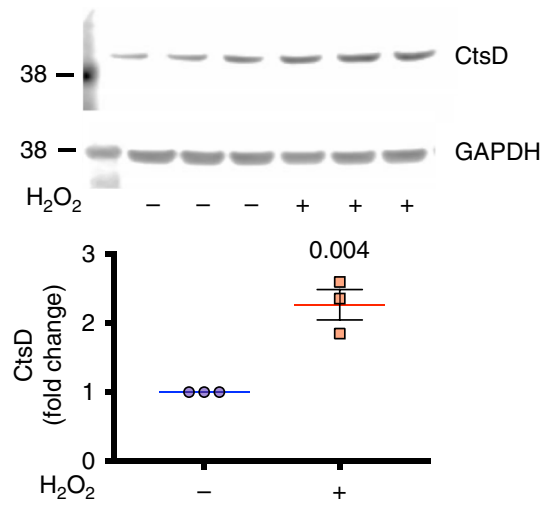

f
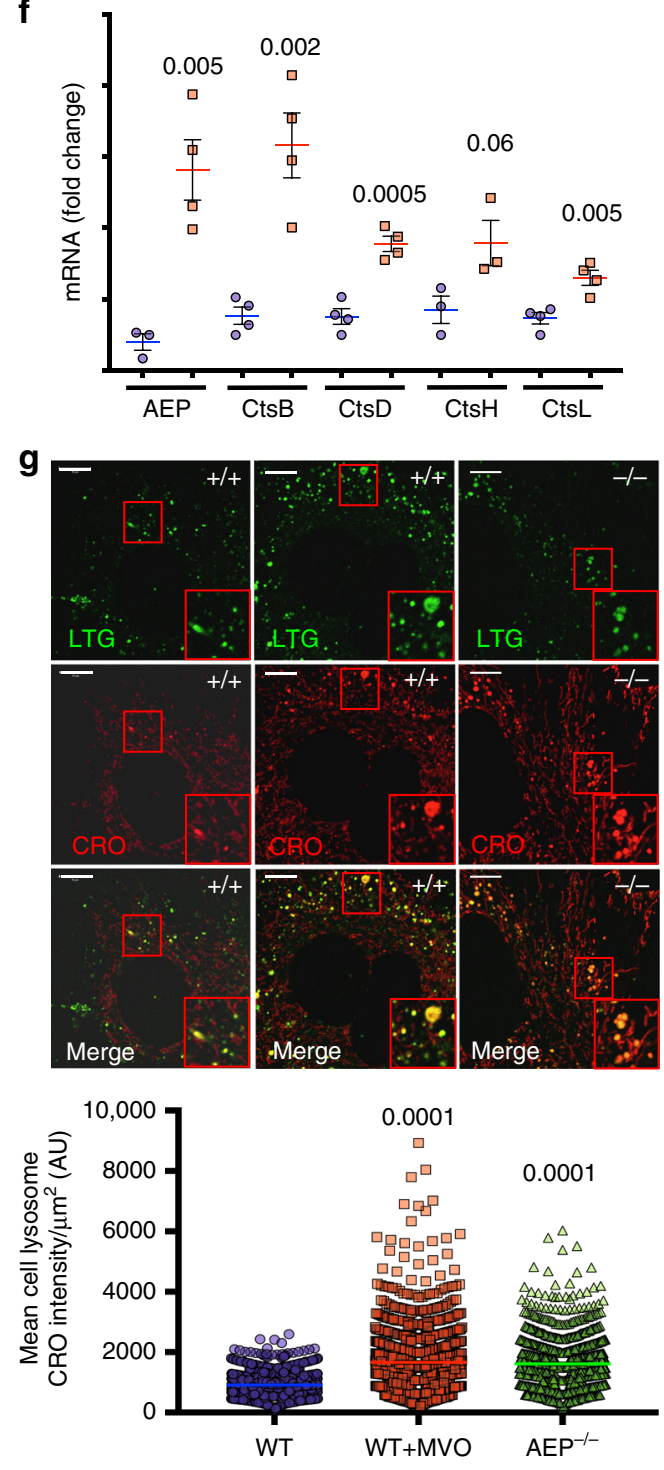

for example through an inhibitory interaction between protein kinase R and STAT3 and/or through suppression of beclin 1 synthesis ${ }^{50-52}$.

The STAT3-driven pathway of increased lysosomal capacity was observed in different cell types and is likely to be a general response to lysosomal stress-induced by protease insufficiency/ substrate accumulation. It may however be particularly relevant in the kidney whose PTC are tasked with reabsorption and processing of a continual macromolecule load present in the 
Fig. 7 Reactive oxygen species: At the nexus between lysosomal hydrolytic capacity and STAT3 phosphorylation. a Superoxide dismutase 1 (SOD1) activity in WT and AEP-/- MEFs. Data are the average \pm SD of $n=3$ biologically independent samples. Statistical significance was calculated using a two-sided unpaired $t$-test. b SOD1 activity in WT MEFs treated for $16 \mathrm{~h}$ with increasing concentrations of MVO26630. Data are the average \pm SD of $n=3$ biologically independent samples. Statistical significance was calculated using a Dunnett's multiple comparison test. c MVO26630-induced (6 h, $50 \mu$ M) STAT3 activation in the presence of increasing concentrations of alpha Phenyl-N-Tert Butyl Nitrone (PBN). Data are the average \pm SD of $n=3$ biologically independent samples. Statistical significance was calculated using a Dunnett's multiple comparison test. d Cellular distribution of TFEB-GFP (upper panels) and STAT3-YFP (lower panels) in HKC-8 WT treated or untreated with $0.2 \mathrm{mM} \mathrm{H}_{2} \mathrm{O}_{2}$ for $16 \mathrm{~h}$. Quantitative data show the nuclear fluorescence intensity per cell ( $n=79$ for TFEB control, $n=99$ for TFEB $+\mathrm{H}_{2} \mathrm{O}_{2}, n=59$ for STAT3 control and $n=58$ for STAT3 $+\mathrm{H}_{2} \mathrm{O}_{2}$ ). Statistical significance was calculated

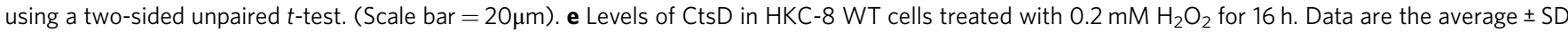
of $n=3$ biologically independent samples. Statistical significance was calculated using a two-sided unpaired $t$-test. $\mathbf{f}$ Quantitative PCR analysis of the mRNA levels encoding several different lysosomal proteases in WT MEFs treated or not with $0.2 \mathrm{mM} \mathrm{H}_{2} \mathrm{O}_{2}$ overnight. Data are the average \pm SD of $n=4$ biologically independent samples for all samples, except for $\mathrm{CtsH}$ where $n=3$. Statistical significance was calculated using a two-sided unpaired $t$-test. $\mathbf{g}$ Live cell imaging of lysosomal production of reactive oxygen species using CellROX Orange (CRO) in AEP-/- or WT MEFs treated or not with $50 \mu M$ MVO26630 for $16 \mathrm{~h}$. LysoTracker Green DND26 (LTG) was used as a lysosomal marker. Data represent the mean CRO intensity per lysosome normalised to lysosomal size in $\mu \mathrm{m}^{2}$ ( $n=1254$ lysosomes for WT MEFs sample, $n=1413$ lysosomes for WT MEFs + MVO sample and $n=1204$ for AEP MEFs). (Scale bar $=10 \mu \mathrm{m})$. Statistical significance was calculated using a Dunnett's multiple comparison test

glomerular ultrafiltrate. Faced with an increased protein load as occurs in proteinuria, PTC likely need to increase lysosomal capacity. Indeed, in focal segmental glomerulosclerosis (FSGS), a model of glomerular injury, proteinuria led not only to increased lysosome protein content but also to an apparently adaptive increase in some lysosomal proteases ${ }^{53}$. A mechanistic basis for this was not elucidated but based on our results it seems likely that proteinuria leads to lysosomal stress, activation of STAT3 in PTC and that this is the basis of increased biosynthesis of lysosomal hydrolases. Activation of STAT3 as a result of lysosomal stress may explain some of the inflammatory features of proteinuric kidney disease. Linked to this, other studies have shown that protein overloaded proximal tubular cells express several vasoactive and inflammatory mediators ${ }^{54}$ and that the Jak/STAT pathway was activated following protein overload of PTC in vitro ${ }^{55}$. With a full complement of lysosomal protease genes available, resolution of lysosomal overload would be expected to extinguish the oxidative stress that drives STAT3 activation and consequently, return hydrolase transcription to normal levels. However, when a critical gene such as AEP is missing and accumulation of target substrates continues, STAT3 activation becomes chronic likely explaining the onset of the severe proliferative kidney disease in AEP-null mice ${ }^{26}$.

Oxidative stress is a common feature of lysosomal storage diseases (LSD) but how these diverse genetic deficiencies lead to oxidative stress is not fully understood ${ }^{43}$ and this is also the case in AEP-deficient cells. However, it is known that loss or mutation of another lysosomal protease, CtsD can lead to congenital neuronal ceroid-lipofuscinosis characterised by accumulation of lipofuscin laden vacuoles and oxidative damage to brain pericytes and other cells ${ }^{16}$. Other studies show that intracranial administration of the pan-lysosomal cysteine protease inhibitor, leupeptin also led to ceroid-lipofuscin accumulation in rat brain ${ }^{56}$. Lipofuscin is a complex undegraded material, rich in protein, lipid, carbohydrates and transition metals, that is associated with the generation of reactive oxygen species ${ }^{57}$. Thus while the details of how oxidative stress-induced by protease/substrate imbalance remain to be clarified, there is clear evidence that lysosomal proteases are non-redundantly required for normal lysosomal homoeostasis and that their loss leads to various forms of lysosomal stress and downstream pathology.

The lysosome-mediated pathway of cell death has been well characterised ${ }^{58}$ but has only recently been shown to be physiologically important. Watson and colleagues have provided striking evidence that post-lactational involution of the mammalian mammary gland is achieved by lysosome-mediated programmed cell death (LM-PCD), dependent on STAT3 activation ${ }^{7,8}$. STAT3 increased expression of CtsB and CtsL and increased uptake of milk fat globules which, upon hydrolysis, released lysosomepermeabilising unsaturated fatty acids which were essential for the induction of LM-PCD. We suggest that the STAT3-driven pathway of lysosomal hydrolase expression may primarily be one for lysosomal adaptation to greater substrate load as shown here. However, as shown by studies in the involuting mammary gland, when combined with perturbations to the lysosomal membrane it can also be used to drive LM-PCD.

In summary, our results shed light on how cells can adjust their lysosomal hydrolytic capacity under conditions of nutritional sufficiency rather than insufficiency which leads to TFEB activation. Communication between the lysosomal lumen and the cytosol appears to depend on lysosomal stress signals rather than lysosomal amino acid levels. Finally, there is great interest in manipulation of the lysosomal system, for example through TFEB activation, to enhance clearance of protein aggregates that can lead to pathology for example in neurodegenerative disease. Our results may suggest additional strategies involving the STAT3 signalling axis and potentially AEP inhibition, which as shown here can lead to extensive adaptational changes that overall, boost lysosome performance.

\section{Methods}

Antibodies. All antibodies used in this manuscript were used a 1:1000 dilution when used for immunoblotting and 1:200 when used for immunofluorescence. Antibodies against P-STAT3 (D3A7), STAT3 (124H6), P-Jak2 (D2E12), Jak2 (3771A), P-4EBP1 (236B4), LC3 (D3U4C) and GAPDH (14C10) were obtained from Cell Signaling. Antibodies against Laminin (ab11575), CtsD (ab75852), CtsB (ab92955), Lamp1 (ab24170) and Fibronectin (ab199056) were purchased from AbCam. Anti-Ki67 (B56) obtained from BD Bioscience, anti-Gns (13044-1-AP) from ProteinTech, anti-TFEB (A303-673A) from Bethyl, anti-YM1 (60130) from Stem Cell Technologies, anti-EGFR (sc-03) from SantaCruz, anti-OSMR $\beta$ (3458100) from R\&D System and anti-SOD1 (3458-100) from BioVision.

Mice and cell culture. WT and $\mathrm{AEP}^{-1-}$ mice on a C57BL/6 background, generated as previously reported ${ }^{59}$, were bred and maintained under pathogen-free conditions under UK Home Office Project License PPL60/3851 and with project approval from the University of Dundee Ethical Review Committee. WT and AEP-null primary murine embryonic fibroblast (MEF) were generated from WT or $\mathrm{AEP}^{-1-}$ mice as previously described ${ }^{60}$ and expanded using complete Dulbecco's modified Eagle medium (DMEM) supplemented with $100 \mathrm{U} / \mathrm{ml}$ penicillin, $100 \mu \mathrm{g} / \mathrm{ml}$ streptomycin, $2 \mathrm{mM}$ L-glutamine, $1 \%$ pyruvate, $1 \%$ non-essential amino acids and $10 \%$ FBS. For protein overload experiments, wildtype MEF were incubated with bovine serum albumin $(30 \mathrm{mg} / \mathrm{ml})$ on coverslips for immunofluorescence experiments or in 6 well plates to measure lysosomal proteolytic activities. HKC-8, a human-derived proximal tubule cell line ${ }^{33}$, were cultured in 1:1 Dulbecco's modified Eagle's-F12 medium supplemented with $100 \mathrm{U} / \mathrm{ml}$ penicillin, $100 \mu \mathrm{g} / \mathrm{ml}$ streptomycin, $2 \mathrm{mM} \mathrm{L}$ glutamine and 5\% FBS. 
a

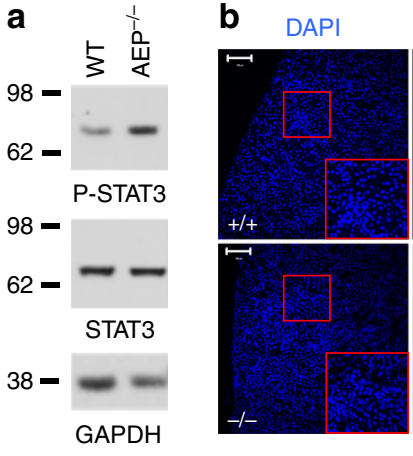

P-STAT3

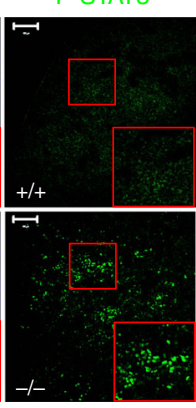

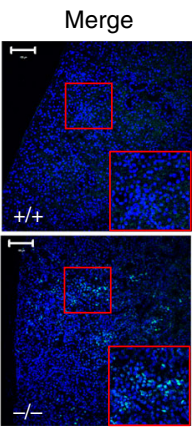

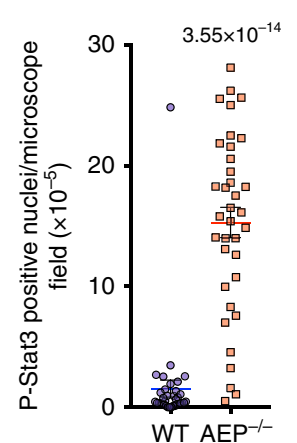

C

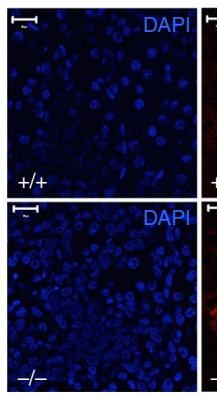

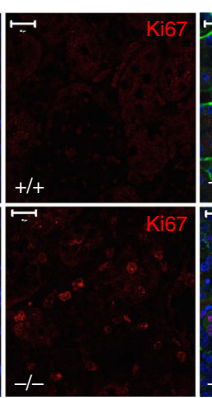

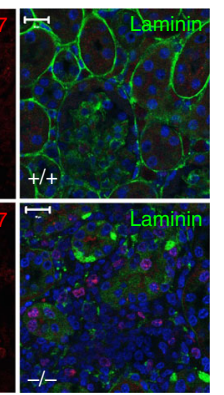

d
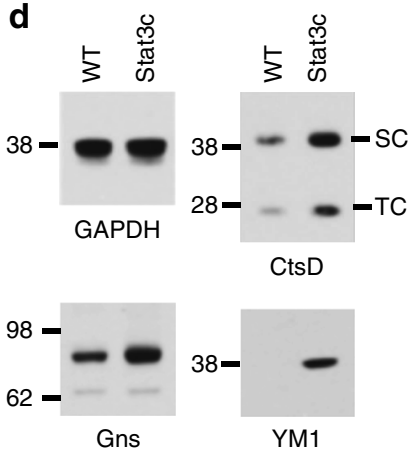

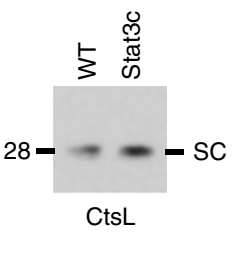

e

PTCs

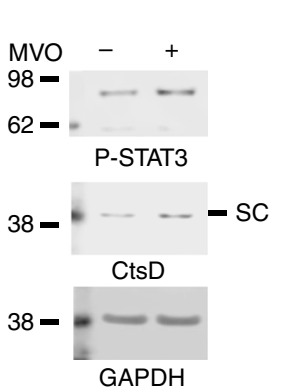

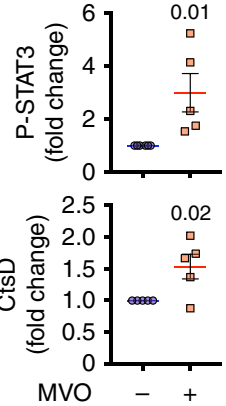

DTCs

Fig. 8 Activation of STAT3 in AEP ${ }^{-/-}$kidney and enhanced hydrolase expression in STAT3c kidneys. a STAT3 and P-STAT3 levels in WT and AEP-/kidneys. b P-STAT3 detected by IF in WT and AEP-/- kidney sections. Right panel shows quantitation of the number of P-STAT3 positive nuclei per area $(n=35$ microscopic fields). (Scale bar $=100 \mu \mathrm{m}$ ). Statistical significance was calculated using a two-sided unpaired $t$-test. c Ki67 and laminin detected by IF in WT and AEP ${ }^{-/-}$kidney sections. (Scale bar $=20 \mu \mathrm{m}$ ). d Similar proteins accumulate in STAT3c and AEP ${ }^{-/-}$kidney. e Levels of P-STAT3 and CtsD in proximal (PTC) and distal (DTC) tubular cells treated or not with $50 \mu \mathrm{M}$ MVO26630 overnight. Data are the average \pm SD of $n=5$ biologically independent samples for PTCs and $n=3$ for DTCs. Statistical significance was calculated using a two-sided unpaired $t$-test

Isolation of human primary proximal (PTC) and distal tubular cells (DTC). Human kidneys cells were isolated from adult kidneys after surgical resection in accordance with the Research Ethics Committee guidelines and approval granted by the NRES Committee East Midlands-Derby (REC reference 13/EM/0311) as previously described ${ }^{61}$. Informed patient consent was obtained as part of this approval. Kidney cortex was minced and digested with $1 \mathrm{mg} / \mathrm{ml}$ collagenase IV for $60 \mathrm{~min}$ at $37^{\circ} \mathrm{C}$ and passed through a $40 \mu \mathrm{m}$ cell strainer. After centrifugation at $300 \times g$, the pellet was resuspended in RPMI and loaded in a two layer Percoll gradient $(50 \%, 24.6 \%)$. Centrifugation at $1000 \times \mathrm{g}$ for $25 \mathrm{~min}$ at $4{ }^{\circ} \mathrm{C}$ produced three separate layers: a top layer containing DTC and a middle layer containing PTC were retrieved and separately washed twice in RPMI at $300 \times g$. DTC were seeded and maintained in DMEM/F-12, GlutaMAX ${ }^{\mathrm{m}}$ supplemented with $100 \mathrm{U} / \mathrm{ml}$ penicillin, $100 \mu \mathrm{g} / \mathrm{ml}$ streptomycin, $2 \mathrm{mM}$ L-glutamine, $10 \% \mathrm{FBS}$ and maintained at $37^{\circ} \mathrm{C}$ in an atmosphere of $5 \% \mathrm{CO}_{2}$. PTC were seeded and maintained in REGM BulletKit media from Lonza supplemented with $100 \mathrm{U} / \mathrm{ml}$ penicillin, $100 \mu \mathrm{g} / \mathrm{ml}$ streptomycin. Cells were used between passage 2 and 3. Phenotypic PTC and DTC confirmation was obtained by electron microscopy.

Transmission electron microscopy. The kidney cortex was cut into small $(1 \mathrm{~mm}$ thick) pieces, and immediately fixed in Karnovsky's fixative (2.5\% glutaraldehyde, $4 \%$ paraformaldehyde in $100 \mathrm{mM}$ cacodylate buffer $\mathrm{pH}$ 7.2). After washing twice in cacodylate buffer and post-fixing in $1 \% \mathrm{OsO}_{4}$ in cacodylate buffer for $60 \mathrm{~min}$, the samples were dehydrated in alcohol, transferred to $100 \%$ propylene oxide and finally incubated overnight on a rotator in $50 \%$ propylene oxide: $50 \%$ Durcupan resin (Sigma). After transfer to $100 \%$ Durcupan resin and polymerisation at $60^{\circ} \mathrm{C}$ overnight, sections (70-100 nm thick) were cut using an ultramicrotome (Leica Ultracut UCT) and stained with 3\% uranyl acetate followed by Reynolds lead citrate ( $1.33 \mathrm{~g}$ lead nitrate, $1.76 \mathrm{~g}$ sodium citrate, $8 \mathrm{ml} 1 \mathrm{M} \mathrm{NaOH}$ in $50 \mathrm{ml}$ water). Grids were imaged on a JEOL 1200 TEX TEM and images were collected on Digital Imaging Plates and scanned on a Ditabis Micron scanner (Pforzheim, Germany).

Enzymatic activity assays. Cell lysates were prepared in $0.2 \mathrm{M} \mathrm{Na}$ citrate buffer $\mathrm{pH} 4.0$ containing $1 \%$ Triton X-100. To measure AEP and CtsB/L activities $20 \mu \mathrm{g}$ of total protein were incubated in $200 \mu \mathrm{l}$ assay buffer $(0.2 \mathrm{M} \mathrm{Na}$ citrate buffer $\mathrm{pH} 4.0$, $1 \mathrm{mM}$ DTT) containing $10 \mu \mathrm{M}$ AEP substrate [Z-Ala-Ala-Asn-7-amino-4-methyl coumarin (AMC)] or $10 \mu \mathrm{M}$ CtsB/L substrate [Z-Phe-Arg-AMC], respectively. AMC release was quantified at $460 \mathrm{~nm}$ in a fluorescence plate reader (Fluorostar
Optima, BMG Labtech), and activities calculated as arbitrary units. CtsD/E activities were measured under the same conditions but using $50 \mu \mathrm{g}$ of cell lysate, $10 \mu \mathrm{M}$ CtsD/E substrate [MCA-Gly-Lys-Pro-Ile-Leu-Phe-Arg-Leu-Lys(Dnp)-D-Arg-

$\mathrm{NH} 2$ ] and MCA release measured at $390 \mathrm{~nm}$. Activities were calculated as arbitrary units.

Superoxide dismutase [Cu-Zn] (SOD1) activity was determined using $20 \mu \mathrm{g}$ of total cell lysate (TBS plus $1 \%$ Triton X-100) from WT or $\mathrm{AEP}^{-1-}$ MEF treated or untreated with different inhibitors using the Superoxide Dismutase Assay Kit following the manufacturer's instructions (ScienCell Research Laboratories).

Immunofluorescence microscopy. Kidneys were removed immediately from culled WT and $\mathrm{AEP}^{-1-}$ mice and fixed in $10 \%$ buffered formalin and embedded in paraffin blocks. Sections ( $5 \mu \mathrm{m}$ thick) were cut, deparaffinised using Histo-Clear and subjected to antigen retrieval using pressure cooking in $\mathrm{Na}$ citrate buffer, $\mathrm{pH}$ 6.0. Sections were permeabilized with $1 \times$ TBS supplemented with $0.025 \%$ Triton $x-$ 100 and blocked with $10 \%$ FBS and 1\% BSA in TBS prior to overnight staining at 4 ${ }^{\circ} \mathrm{C}$ with the following primary antibodies prepared in TBS, $1 \%$ BSA: rabbit anti-PSTAT3, mouse anti-Ki67, rabbit anti-laminin, rabbit anti-CtsD, rabbit anti-Gns, rabbit-anti-TFEB, rabbit anti-P-4EBP1 (dilution 1:200). After washing in TBS $0.025 \%$ Triton X-100 twice, the slides were incubated for $1 \mathrm{~h}$ with the appropriate Alexa-488- or Alexa 594-conjugated secondary antibody (Invitrogen, dilution 1:500) in TBS with 1\% BSA. The slides were finally washed three times in TBS and mounted under coverslips using Vectashield mounting medium containing DAPI (Vector Laboratories) and viewed using an LSM 700 confocal microscope (Carl Zeiss).

WT and $\mathrm{AEP}^{-1-}$ MEF $\left(5 \times 10^{4}\right.$ cells/coverslip) were fixed with $4 \%$ paraformaldehyde in PBS at room temperature for $20 \mathrm{~min}$ or with $100 \%$ methanol at $-20^{\circ} \mathrm{C}$ for $10 \mathrm{~min}$. The cells were washed three times with PBS, permeabilized with $0.2 \%$ Triton X-100 in PBS for $20 \mathrm{~min}$ at room temperature, washed $3 \times$ with PBS and then incubated for $20 \mathrm{~min}$ at room temperature in blocking buffer $(1 \%$ BSA in TBS). The cells were stained using a 1:200 dilution of primary antibody in blocking buffer for $45 \mathrm{~min}$ at room temperature (rabbit anti-TFEB, rabbit anti-LC3 or rabbit anti-P-STAT3), washed three times with PBS and stained with a 1:500 dilution of the Alexa-488-conjugated secondary antibody (Invitrogen) for another $45 \mathrm{~min}$ at room temperature. Coverslips were washed three times with PBS $1 \times$ and mounted using Vectashield mounting medium containing DAPI (Vector Laboratories) and viewed using an LSM 700 confocal microscope (Carl Zeiss). 

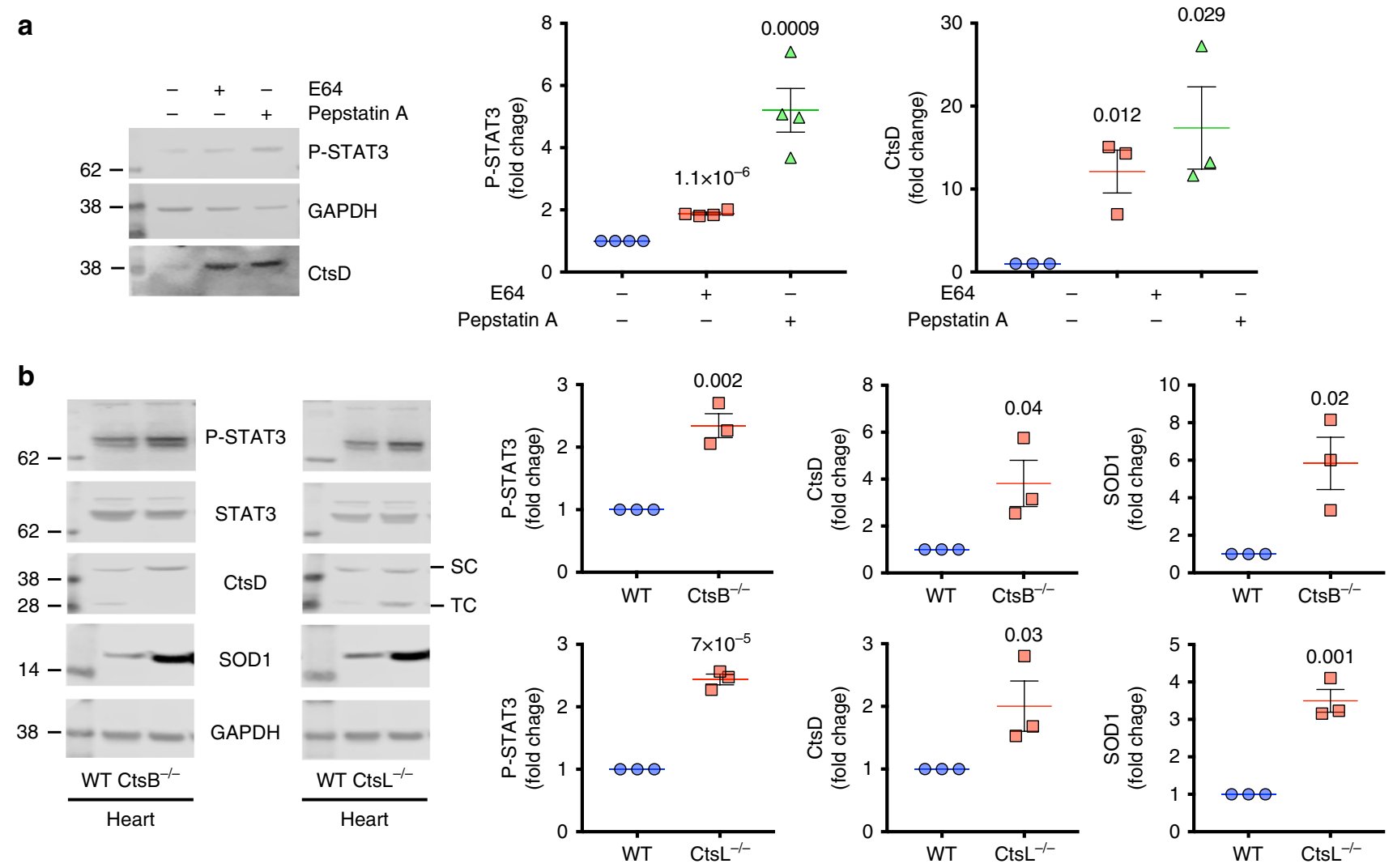

Fig. 9 Chronic or acute inhibition of cysteine or aspartyl cathepsins lead to a similar response. a Levels of P-STAT3 and CtsD in WT MEFs treated or not with $10 \mu \mathrm{M} \mathrm{E64}$ or $5 \mu \mathrm{M}$ Pepstatin A for $72 \mathrm{~h}$. Data are the average \pm SD of $n=4$ biologically independent samples for P-STAT3 and $n=3$ biologically independent samples for CtsD. Statistical significance was calculated using a two-sided unpaired $t$-test. $\mathbf{b}$ Levels of P-STAT3, STAT3, CtsD and SOD1 in $\mathrm{CtsB}^{-/-}$heart or $\mathrm{CtsL}^{-/-}$heart compared to WT heart. Data are the average $\pm \mathrm{SD}$ of $n=3$ biologically independent samples. Statistical significance was calculated using a two-sided unpaired $t$-test

STAT3-YFP and TFEB-GFP cellular distribution in HKC-8 cells. The TFEB-GFP construct was purchased from Acris Antibodies. STAT3-YFP was cloned into pcDNA5. HKC-8 cells $\left(5 \times 10^{4}\right.$ cells/coverslip) were transfected with STAT3-YFP or TFEB-GFP using polyethylenimine $(\mathrm{PEI})^{62}$ and $24 \mathrm{~h}$ later cells were treated as indicated in the figure legends. After fixation with $4 \%$ paraformaldehyde in PBS at room temperature for $20 \mathrm{~min}$ the coverslips were washed three times with PBS $1 \times$ and mounted using Vectashield mounting medium containing DAPI (Vector Laboratories) and viewed using an LSM 700 confocal microscope (Carl Zeiss).

TFEB knockout generation in HKC-8 cells. Two different TFEB knockouts were generated by targeting TFEB exon 1 or exon 2 . The sense guides (exon 1: GCATGCAGCTCATGCGGGAGC; exon 2: GTGCCCCAGCAGCATCCCCAG) were cloned into the pBABED puro U6 and the antisense guides were cloned into the Cas9 D10A vector pX355 (exon 1: GCAGCCCGATGCGTGACGCCA; exon 2: GGCTTCGGGGAACCTTGGGC)

HKC- 8 cells $\left(10^{6}\right.$ cells $/ 10 \mathrm{~cm}$ dish) were transfected with the sense and antisense guide plasmids targeting exon 1 or exon 2 of the TFEB gene using PEI. Twentyfour hours later the media was replaced with media supplemented with $2 \mu \mathrm{g} / \mathrm{ml}$ puromycin. After $48 \mathrm{~h}$ of puromycin selection transfected cells were expanded and individual clones were isolated and tested for TFEB expression by western blotting.

Jak2 knockdown in HKC-8 cells. A set of four Jak2-siRNAs were purchased from Dharmacon and tested individually to determine levels of knockdown achieved. The siRNAs providing the highest level of Jak2 knockdown (siRNA \#6: GAAUUGUAACUGUCCAUAA and siRNA \#7: GAAUUUAUGCGAAUGAUUG) were subsequently used in all the experiments.

HKC- 8 cells $\left(10^{4}\right.$ cells per well $)$ in a 96 well plate were transfected with Jak2siRNAs or non-targeting siRNA (UGGUUUACAUGUCGACUAA) (Dharmacon) using DharmaFect 1 transfection reagent (Dharmacon) following the manufacturer's instructions. Forty-eight hours later cells were treated as indicated and samples prepared for SDS-PAGE analysis.

STAT3 knockdown and rescue in HKC-8 cells. A set of four STAT3-siRNAs were purchased from Dharmacon and tested individually to determine levels of knockdown achieved. The siRNAs providing the highest level of STAT3 knockdown (siRNA \#8: CAACAUGUCAUUUGCUGAA and siRNA \#10: CAACAGAUUGCCUGCAUUG) were subsequently used in all the experiments. HKC8 cells $\left(10^{4}\right.$ cells per well $)$ in a 96 well plate were transfected with STAT3-siRNAs or non-targeting siRNA (UGGUUUACAUGUCGACUAA) (Dharmacon) using DharmaFect 1 transfection reagent (Dharmacon) following the manufacturer's instructions. Forty-eight hours later cells were treated as indicated and samples prepared for SDS-PAGE analysis

To generate a form of STAT3 refractory to siRNA knockdown, we fused it to GFP to distinguish it from endogenous STAT3 (mSTAT3-GFP) and introduced mutations into the target sequence for siRNA \#8 (see above) using the following primers $5^{\prime}$-GCAGCTGAACAACATGTCATTTGCTGAAATCATCATG- ${ }^{\prime}$ ' and 5'-CATGATGATTTCAGCAAATGACATGTTGTTCAGCTGC-3'. HKC-8 cells were transiently transfected using PEI and GFP-positive cells were enriched by FACS using an Influx cell sorter (Becton Dickinson). Cells were distinguished from debris based on FSC-H vs SSD-H and single cells on the basis of FSC-A vs FSC-W measurements. GFP-positive cells were identified based on background fluorescence levels in the parental cell line negative control. Then, sorted cells were expanded for $48 \mathrm{~h}$ in the presence of G418 to maintain plasmid expression prior to siRNA (\#8 or non-targeting) transfection. Knockdown of endogenous STAT3 versus mSTAT3-GFP was assessed by western blotting and STAT3 activation by MVO-treatment was performed and analysed as previously described

STAT3 ChIP-PCR. Anti-STAT3 ChIP was carried out as previously reported ${ }^{63}$. Briefly, WT and $\mathrm{AEP}^{-1-}$ MEFs were grown to $70 \%$ confluency, crosslinked with $1 \%$ formaldehyde for $10 \mathrm{~min}$ at RT. Excess formaldehyde was blocked with $0.125 \mathrm{M}$ glycine and the cells washed with ice-cold PBS several times after which the cells were scraped off in $2 \mathrm{ml}$ of PBS containing protease inhibitors and collected by centrifugation at $500 \times \mathrm{g}$ for $10 \mathrm{~min}$ at $4{ }^{\circ} \mathrm{C}$. Pellets were resuspended in lysis buffer (1\% SDS, $10 \mathrm{mM}$ EDTA, $50 \mathrm{mM}$ Tris- $\mathrm{HCl}(\mathrm{pH} 8.1$ ) and protease inhibitors) and sonicated three times for $5 \mathrm{~min}$ in a BioRuptor RTH ( $30 \mathrm{~s}$ on $/ 30 \mathrm{~s}$ off). Samples were then diluted 1:10 in ChIP dilution buffer (1\% Triton X-100, 2 mM EDTA, $150 \mathrm{mM} \mathrm{NaCl}, 20 \mathrm{mM}$ Tris- $\mathrm{HCl}(\mathrm{pH} 8.1)$ ) and anti-STAT3 antibody was added to the samples (Cell Signaling, 124H6) and incubated overnight at $4{ }^{\circ} \mathrm{C}$. Finally immunoprecipitated chromatin was purified using the iPure Kit (Diagenode) following the manufacturer's instructions. 
The samples were then tested for the presence of the promoter regions of genes of interest by PCR using the following primers:

STAT3 (forward: GGTGACACCTGGGGACCGCCTAAG; reverse:

AAAAACGCCTCTAGGAGAGAAGGCG),

AEP (forward: ATGAGTTGGCCCTGTTTTGGGTCC; reverse:

TCTGCCTTGGCCTATGATCACAAC),

CtsL (forward: AAAACAGTCTCCATCGTTCTCAAC; reverse:

GGCCTGCTGTGGAATTTCCACCTC),

CtsD (forward: AGAGTGAAGTAGTTAAGACCCAAG; reverse:

TACCAGGATGTGGTCTTTGTCTGA),

Gns (forward: GGAAGGTGCGTGAGCACGCAAAAC; reverse:

GGACAGAGCACCGGAGATGAAGAC),

Man2b2 (forward: GATAGAGAGGGATCTGAGCTTGCT; reverse:

CAGAATCAAATCTGGGGTCTTGGA),

Naga (forward: GTTAAGAGCAAGGTTTCAGGTGGA; reverse:

GTTCTGCGCGTTCCCGAGGCGCGC),

Plbd2 (forward: TGCAGAGGACTTGAGTTCGAATTC; reverse:

CTCAGCATCCTTCCCTCCCAAGGA).

The sequence of the primers used as negative controls was: (forward:

ATGGTTGCCACTGGGGATCT; reverse: TGCCAAAGCCTAGGGGAAGA)

Electrophoretically separated PCR products were quantified with ImageJ and normalised to input samples.

Generation of ${ }^{13} \mathbf{C}_{6}$-Lysine SILAM mouse colony. An in-house breeding colony of C57BL/6 ${ }^{13} \mathrm{C}_{6}$-Lysine 'SILAM' labelled mice was generated using specialised ${ }^{13} \mathrm{C}_{6}$-Lysine mouse diet (Silantes, Martinsried, Germany) following the breeding programme previously described by Krüger et al. ${ }^{29}$ Labelling efficiency was monitored for brain, liver and skin tissues from 19 weeks old F0 (fed ${ }^{13} \mathrm{C}_{6}$-Lysine mouse diet for 16 weeks), 8-week-old F1 and 8-week-old F2 animals. Labelling efficiencies for all confidently ( $1 \%$ false discovery rate) assigned peptides were calculated as: heavy_peptide_area /(heavy_peptide_area + light_peptide_area). For all tested samples, $\sim 90 \%$ of the peptides were $>90 \%{ }^{13} \mathrm{C}_{6}$-Lysine-labelled at the $\mathrm{F} 2$ generation. Tissues used for this study were acquired from F4 C57BL/6 ${ }^{13} \mathrm{C}_{6}$-Lysine SILAM females.

Kidney lysosome SILAC-based mass spectrometry. Kidney lysosomes from Llysine- ${ }^{13} \mathrm{C}_{6}$ labelled WT mice and unlabelled WT or $\mathrm{AEP}^{-1-}$ mice were prepared as previously described ${ }^{26} .60 \mu \mathrm{g}$ of unlabelled WT or $\mathrm{AEP}^{-1-}$ kidney lysosomal proteins were combined in a $1: 1$ ratio with $60 \mu \mathrm{g}$ of $\mathrm{L}-$-lysine- ${ }^{13} \mathrm{C}_{6}$ labelled WT kidney lysosomal proteins. Protein samples were prepared for LC-MS/MS using the FASP protocol as previously described ${ }^{64}$. Briefly, the protein mixture was denatured at $95^{\circ} \mathrm{C}$ for $5 \mathrm{~min}$ in SDS-lysis buffer (4\% (w/v) SDS, $100 \mathrm{mM}$ Tris- $\mathrm{HCl} \mathrm{pH}$ $7.6,0.1 \mathrm{mM} \mathrm{DTT}$ ) and the samples were mixed with $200 \mu \mathrm{l}$ of $8 \mathrm{M}$ urea in 0.1 Tris- $\mathrm{HCl} \mathrm{pH} 8.5$ (UA buffer) in a Microcon YM-10 filter unit and centrifuged at $17,000 \times g$ for $40 \mathrm{~min}$. Samples were washed again with another $200 \mu \mathrm{l}$ of UA buffer and then treated with $100 \mu$ of $0.05 \mathrm{M}$ iodoacetamide in UA buffer for $20 \mathrm{~min}$ at room temperature. After three further washes with $100 \mu \mathrm{l} \mathrm{UA}$ buffer and three times with $0.05 \mathrm{M} \mathrm{NH}_{4} \mathrm{CO}_{3}$ (ABC buffer) centrifuging each time at $14,000 \times \mathrm{g}$ for 15 min and discarding the flow through, the protein mixture was digested overnight at $37^{\circ} \mathrm{C}$ in the YM-10 filter unit using $40 \mu \mathrm{l}$ of $0.4 \mu \mathrm{g} / \mu \mathrm{l}$ trypsin in ABC buffer. The samples containing the tryptic peptides were collected by centrifugation at $14,000 \times g$ for $40 \mathrm{~min}$ and $50 \mu \mathrm{l}$ of $0.5 \mathrm{M} \mathrm{NaCl}$ was added to the YM-10 filters followed by centrifugation for another $20 \mathrm{~min}$ at $14,000 \times g$. Tryptic peptides were finally desalted using a C18-SD cartridge, treated with $0.1 \%$ trifluoroacetic acid and eluted in $70 \%$ acetonitrile.

MEF SILAC-based mass spectrometry. MEF were cultured for at least 10 days in RPMI without arginine and lysine (Biosera) containing $10 \%$ dialysed FCS (Hyclone), $100 \mathrm{U} / \mathrm{ml}$ penicillin, $100 \mu \mathrm{g} / \mathrm{ml}$ streptomycin, $2 \mathrm{mM}$ L-glutamine, $1 \%$ pyruvate, $1 \%$ non-essential amino acids and supplemented with $84 \mathrm{mg} / \mathrm{l}$ of Larginine (Sigma) and $40 \mathrm{mg} / \mathrm{l}$ of L-lysine (Sigma) for the 'light' medium or $106 \mathrm{mg} /$ $\mathrm{ml}$ of $\mathrm{L}$-arginine ${ }^{-13} \mathrm{C}_{6}{ }^{-15} \mathrm{~N}_{4}$ and $50 \mathrm{mg} / \mathrm{ml}$ of L-lysine- ${ }^{13} \mathrm{C}_{6}$ for the 'heavy' medium. Equal numbers of cells were mixed and cytoplasmic, membrane and nuclear fractions were obtained using a subcellular protein fractionation kit for cultured cells (Thermo) according to the manufacturer's instructions. $100 \mu \mathrm{g}$ of protein was size-fractionated by SDS-PAGE using 4-12\% NuPAGE gels (Invitrogen) in MES running buffer, stained using SimplyBlue Safe Stain (Invitrogen) and destained using miliQ water. The gels were cut into $15 \times 0.5 \mathrm{~cm}$ slices using a clean scalpel and cut into $1 \mathrm{~mm}$ cubes. After washing with $50 \%$ acetonitrile/water, $0.1 \mathrm{M}$ $\mathrm{NH}_{4} \mathrm{CO}_{3}$ and $50 \%$ acetonitrile in $50 \mathrm{mM} \mathrm{NH}_{4} \mathrm{CO}_{3}(0.5 \mathrm{ml})$ the samples were treated with $75 \mu \mathrm{l}$ of $10 \mathrm{mM}$ DTT in $0.1 \mathrm{M} \mathrm{NH}_{4} \mathrm{CO}_{3}$ and incubated for $20 \mathrm{~min}$ at $37^{\circ} \mathrm{C}$. The liquid was removed and proteins in the gel pieces were carboxymethylated with $50 \mathrm{mM}$ iodoacetamide in $0.1 \mathrm{M} \mathrm{NH}_{4} \mathrm{CO}_{3}$ for $20 \mathrm{~min}$ at room temperature in the dark. The gel pieces were washed with $50 \%$ acetonitrile in $50 \mathrm{mM} \mathrm{NH} \mathrm{CO}_{3}$, shrunk using $0.3 \mathrm{ml}$ acetonitrile per piece for $15 \mathrm{~min}$ and after removal of the supernatant, dried using a Speed-Vac lyophiliser. The protein content of each piece was digested with $30 \mu \mathrm{l} 5 \mu \mathrm{g} / \mathrm{ml}$ of trypsin in $25 \mathrm{mM}$ triethylammonium bicarbonate at $30^{\circ} \mathrm{C}$ overnight. An equal volume of acetonitrile was added and the mixture was shaken at $30^{\circ} \mathrm{C}$ for $15 \mathrm{~min}$. The supernatants, containing the tryptic peptide mixture from each sample, were frozen in liquid nitrogen and dried using a Speed-Vac. Meanwhile, the gel pieces were treated with $100 \mu$ of $50 \%$ acetonitrile, $2.5 \%$ formic acid in water and shaken for $10 \mathrm{~min}$ at $30^{\circ} \mathrm{C}$. This second supernatant was combined with the first, frozen down in liquid nitrogen and then dried in a Speed-Vac.

Mass spectrometry. A nanoflow liquid chromatograph (Ultimate 3000 RSLCnano system, Thermo) coupled to a LTQ Orbitrap Velos Pro mass spectrometer (Thermo) was used to analyse the protein digests. Samples, usually $10 \mu \mathrm{l}$, were loaded onto a C18 trap column and washed with $0.1 \%$ formic acid. The peptides were resolved using a gradient ( $130 \mathrm{~min}, 0.3 \mu \mathrm{l} / \mathrm{min})$ of buffer A ( $0.1 \%$ formic acid) and buffer B ( $80 \%$ acetonitrile in $0.08 \%$ formic acid): $2 \%$ buffer B for $4 \mathrm{~min}$ followed by $2-40 \%$ buffer B for $64 \mathrm{~min}, 40-98 \%$ buffer B for $2 \mathrm{~min}, 98 \%$ buffer B for $15 \mathrm{~min}, 98-2 \%$ buffer B for $1 \mathrm{~min}$ and $2 \%$ buffer B for $44 \mathrm{~min}$. Peptides, initially trapped on an Acclaim PepMap $100 \mathrm{C} 18$ column $(100 \mu \mathrm{m} \times 2 \mathrm{~cm}$, Thermo) were then separated on an Easy-Spray PepMap RSLC C18 column $(75 \mu \mathrm{m} \times 50 \mathrm{~cm}$, Thermo), and finally transferred to the LTQ Orbitrap Velos Pro via an Easy-Spray source set at $50^{\circ} \mathrm{C}$ and a source voltage of $1.9 \mathrm{kV}$. For the identification of peptides, a top 15 method (FT-MS plus 15 IT-MS/MS, 100 min acquisition) consisting of full scans and mass range $(\mathrm{m} / \mathrm{z})$ between 335 and 1800 was used. The Orbitrap was operated in a profile mode, resolution of 60,000 with a lock mass set at 445.120024 and a max fill time of $500 \mathrm{~ms}$. LTQ was operated in a centroid mode with isolation width $=2.00(\mathrm{~m} / \mathrm{z})$, normalised collision energy $=35.0$, activation time $=10.0 \mathrm{~ms}$ and max fill time of $100 \mathrm{~ms}$.

Mass spectrometry data analysis. LTQ Orbitrap Velos Pro.RAW files were analysed, and peptides and proteins quantified using MaxQuant ${ }^{65}$, using the builtin search engine Andromeda ${ }^{66}$. All settings were set as default, except for the minimal peptide length of 5 , and Andromeda search engine was configured for the UniProt Mus musculus protein database (release date: 2013_10). Peptide and protein ratios only quantified in at least two out of the three replicates were considered, and the $p$-values were determined by Student's $t$ test and corrected for multiple testing using the Benjamini-Hochberg procedure ${ }^{65}$.

Sample preparation and immunoblotting. Tissue samples were prepared using a 7-ml dounce tissue grinder (Wheaton, USA) in complete RIPA buffer (RIPA buffer (Thermo) supplemented with $1 \mathrm{mM}$ sodium orthovanadate, $10 \mathrm{mM}$ sodium fluoride and 1 protease inhibitor tablet (Roche)). WT and AEP ${ }^{-1-}$ MEFs $\left(2 \times 10^{5}\right.$ cells) treated or untreated with the different inhibitors were washed once with icecold PBS and lysed using complete RIPA buffer. Tissue and cell lysates were centrifuged at $20,000 \times g$ for 10 min and equal amounts of protein loaded on $4-12 \%$ NuPAGE gels (Invitrogen) and then transferred onto nitrocellulose membranes (Amersham). Membranes were probed with the following antibodies: rabbit anti-PSTAT3, mouse anti-STAT3, rabbit anti-GAPDH and rabbit anti-LC3 (Cell Signaling), rabbit anti-Ym1 (StemCell Technologies), rabbit anti-EGFR (SantaCruz), rabbit anti-Lamp1, rabbit anti-CtsD, rabbit anti-Fn1 and anti-CtsB (abCam), rabbit anti-TFEB (Bethyl) and rabbit anti-Gns (ProteinTech) and goat anti-OSMR $\beta$ (R\&D Systems). Sheep anti-AEP was obtained as described previously ${ }^{32}$ and sheep anti-CtsL and CtsH were generous gifts from Drs Tina Zavašnik-Bergant and Janko Kos (Jožef Stefan Institute, Ljubljana, Slovenia)

In vivo cathepsin $\mathbf{L}$ activity assay. WT and $\mathrm{AEP}^{-/-} \mathrm{MEF}\left(5 \times 10^{4}\right.$ cells $)$ were grown on coverslips and incubated with Magic Red cathepsin L (Immunochemistry Technologies) for $60 \mathrm{~min}$ at $37^{\circ} \mathrm{C}$ following the manufacturer's instructions. The cells were then fixed with $4 \%$ paraformaldehyde in PBS, mounted using Vectashield mounting medium containing DAPI (Vector Laboratories) and images were obtained using a LSM 700 confocal microscope (Carl Zeiss).

Lysosomal degradation of DQ-BSA. WT $3 \mathrm{~T} 3$ cells $\left(5 \times 10^{4}\right.$ cells $)$ were grown on coverslips and treated with MVO $50 \mu \mathrm{M}$ for $16 \mathrm{~h}$. Then, cells were incubated with $10 \mu \mathrm{g} / \mathrm{ml}$ of DQ-BSA (Invitrogen) or $50 \mu \mathrm{g} / \mathrm{ml}$ of FiTC-BSA for $2 \mathrm{~h}$ at $37^{\circ} \mathrm{C}$, washed twice with PBS, then fixed with $4 \%$ paraformaldehyde in PBS for $20 \mathrm{~min}$ at room temperature, mounted using Vectashield mounting medium with DAPI (Vector Laboratories) and images were obtained using an LSM 700 confocal microscope (Carl Zeiss).

Onconstatin $M$ receptor $\beta$ (OSMR $\beta)$ and epithelial growth factor receptor (EGFR) degradation assay. Total cell extracts from WT cells treated or not with $50 \mu \mathrm{M}$ MVO26630 and $\mathrm{AEP}^{-1-} \mathrm{MEF}\left(2 \times 10^{5}\right.$ cells $)$, incubated as indicated with oncostatin M $25 \mathrm{ng} / \mathrm{ml}$ (R\&D Systems) or epithelial growth factor $100 \mathrm{ng} / \mathrm{ml}$ and cycloheximide $(10 \mu \mathrm{g} / \mathrm{ml})$, were prepared in complete RIPA buffer and equal amounts of protein were separated by SDS-PAGE and subjected to direct Western blotting using anti-OSMR $\beta$ or anti-EGFR as described above.

In-cell reactive oxygen species determination. $\mathrm{WT}$ and $\mathrm{AEP}^{-1-} \mathrm{MEF}$ grown on FluoroDish dishes (World Precision Instruments Inc.) treated as indicated with the different inhibitors were incubated with $100 \mathrm{nM}$ LysoTracker Green DND26 (Invitrogen) for $2 \mathrm{~h}$ at $37^{\circ} \mathrm{C}$ and then with $2.5 \mu \mathrm{M}$ CellROX Orange (Invitrogen) for $30 \mathrm{~min}$ at $37^{\circ} \mathrm{C}$. The cells were washed twice with PBS, fresh complete DMEM 
was added to the dishes and live cell imaging carried out at $37^{\circ} \mathrm{C}$ and $5 \% \mathrm{CO}_{2}$ using a LSM 700 confocal microscope (Carl Zeiss). CellROX Orange intensity overlapping with LysoTracker Green DND26 positive structures was quantified using the Volocity 3D Image Analysis Software (PerkinElmer). The mean intensity of CellROX Orange per LysoTracker Green DND26 positive structure, normalised for lysosomal size $\left(\mu \mathrm{m}^{2}\right)$ and per cell was calculated and the standard error calculated using 15 cells per experimental condition.

\section{Semi-quantitative reverse transcription-polymerase chain reaction (RT-PCR).} Total RNA from WT MEF, treated or untreated with $50 \mu \mathrm{M}$ MVO26630 overnight, and $\mathrm{AEP}^{-1-} \mathrm{MEF}$, WT and STAT3c 3T3s was purified using RNeasy mini kit (Qiagen) following the manufacturer's instructions. Total RNA concentration was determined using a NanoDrop 1000 spectrophotometer (Thermo) and $1 \mu \mathrm{g}$ of total RNA per sample was used to synthesise cDNA using the qScript Flex cDNA kit (Quanta Biosciences) according to the manufacturer's conditions. Finally, the mRNA expression levels of different lysosomal proteases were determined by PCR, quantified using ImageJ (imagej. nih.gov) and normalised using $\beta$-actin or Tubulin 5 as loading control. The following primers were used: $\beta$-actin: forward (ATCATGTTTGAGACCTTCAACA), reverse (CATCTCCTGCTCGAAGTCTA); Tubb5: forward (TGTACTATAATGAAGCCACAGGTGG), reverse (CAGTAG GTCTCATCCGTGTTCTCAA); AEP: forward (ATGACCTGGAGAGTGGCTG), reverse (CGTTGATGTCGTCGGGCA); CtsB: forward (GGGTACTTAGGAG TGCACGG), reverse (CCAAATGCCCAACAAGAGCC); CtsD: forward (TCAGG AAGCCTCTCTGGGTA), reverse (CTGCAGCTCCTTCACCTCTT); CtsH: forward (ATGACGAGGCTGCAATGGTT), reverse (TCCCTTGGTCTGCCAATC AG); CtsL: forward (ATGGCACGAATGAGGAAGAG), reverse

(GAAAAAGCCTCCCCTTCTTG) and CtsZ: forward (CAGCGGATCTCCCCAA GAAT), reverse (GTCCTTGGCCTGGTAGTTGT).

Total RNA from HKC-8 WT or TFEB KOs was purified using RNeasy mini kit (Qiagen) following the manufacturer's instructions. Total RNA concentration was determined using a NanoDrop 1000 spectrophotometer (Thermo) and $1 \mu \mathrm{g}$ of total RNA per sample was used to synthesise cDNA using the qScript Flex cDNA kit (Quanta Biosciences) according to the manufacturer's conditions. Finally, the mRNA expression levels of different lysosomal proteases were determined by PCR, quantified using ImageJ (imagej. nih.gov) and normalised using $\beta$-actin as loading control. The following primers were used: $\beta$-actin: forward (GGGGTGTTGAAGGTCTCAAA), reverse (GGCATCCTCACCCTGAAGTA); CtsB: forward (CGCTTTCCATTCCTGGGTCTCTG), reverse

(ACAGGCCATGTGAGCCACCG); CtsD: forward

(CCCACACACACCCACACACTCG), reverse (CCAGGGAGGGGAAAACCACAGA).

HKC-8 WT AND TFEB KOs Starvation. Total cell extracts from HKC-8 WT and TFEB KO cells starved or not for $4 \mathrm{~h}$ in $\mathrm{HBSS}$ at $37^{\circ} \mathrm{C}$ and $10 \% \mathrm{CO}_{2}$ were prepared in complete RIPA buffer and equal amounts of protein were separated by SDSPAGE and subjected to direct Western blotting using anti-LC3 or anti-CtsB as described above.

GO analysis. ClueGo ${ }^{66}$ within the Cytoscape software ${ }^{67}$ was used to find statistically over-represented gene ontology (GO) categories. The identified proteins from the kidney lysosomal SILAC-based mass spectrometry analysis were used as a test dataset and a reference set of GO annotations for the mouse proteome was used in order to carry out the GO enrichment analysis. Analyses were done using a right-sided hypergeometric test and all GO terms corresponding to cellular location or molecular function with $p<0.005$ were selected.

\section{Data availability}

The authors declare that all primary data are available in main or Supplementary figures. The mass spectrometry proteomics data have been deposited to the ProteomeXchange Consortium via the PRIDE partner repository with the dataset identifier PXD011473. All other data that support the findings of this study are available from the corresponding authors upon reasonable request

Received: 6 August 2018 Accepted: 18 November 2018 Published online: 17 December 2018

\section{References}

1. Sepulveda, F. E. et al. Critical role for asparagine endopeptidase in endocytic Toll-like receptor signaling in dendritic cells. Immunity 31, 737-748 (2009).

2. Ewald, S. et al. The ectodomain of Toll-like receptor 9 is cleaved to generate a functional receptor. Nature 456, 658-662 (2008).

3. Settembre, C. et al. A lysosome-to-nucleus signalling mechanism senses and regulates the lysosome via mTOR and TFEB. EMBO J. 31, 1095-1108 (2012).
4. Sabatini, D. M. Twenty-five years of mTOR: Uncovering the link from nutrients to growth. Proc. Natl Acad. Sci. USA 114, 11818-11825 (2017)

5. Colbert, J. D., Matthews, S. P., Miller, G. \& Watts, C. Diverse regulatory roles for lysosomal proteases in the immune response. Eur. J. Immunol. 39, 2955-2965 (2009).

6. Bird, P. I., Trapani, J. A. \& Villadangos, J. A. Endolysosomal proteases and their inhibitors in immunity. Nat. Rev. Immunol. 9, 871-882 (2009).

7. Kreuzaler, P. A. et al. Stat3 controls lysosomal-mediated cell death in vivo. Nat. Cell Biol. 13, 303-309 (2011).

8. Sargeant, T. J. et al. Stat3 controls cell death during mammary gland involution by regulating uptake of milk fat globules and lysosomal membrane permeabilization. Nat. Cell Biol. 16, 1057-1068 (2014).

9. Luzio, J. P., Hackmann, Y., Dieckmann, N. M. G. \& Griffiths, G. M. The biogenesis of lysosomes and lysosome-related organelles. Cold Spring Harb. Perspect. Biol. 6, a016840 (2014).

10. Shen, H.-M. \& Mizushima, N. At the end of the autophagic road: an emerging understanding of lysosomal functions in autophagy. Trends Biochem. Sci. 39, 61-71 (2014).

11. Sardiello, M. et al. A gene network regulating lysosomal biogenesis and function. Science 325, 473-477 (2009).

12. Ballabio, A. The awesome lysosome. EMBO Mol. Med. 8, 73-76 (2016).

13. Settembre, C. et al. TFEB links autophagy to lysosomal biogenesis. Science 332, 1429-1433 (2011)

14. Li, Y. et al. Protein kinase C controls lysosome biogenesis independently of mTORC1. Nat. Cell Biol. 18, 1065-771-26 (2016).

15. Spira, D. et al. Cell type-specific functions of the lysosomal protease cathepsin $\mathrm{L}$ in the heart. J. Biol. Chem. 282, 37045-37052 (2007).

16. Koike, M. et al. Cathepsin D deficiency induces lysosomal storage with ceroid lipofuscin in mouse CNS neurons. J. Neurosci. 20, 6898-6906 (2000).

17. Reiser, J., Adair, B. \& Reinheckel, T. Specialized roles for cysteine cathepsins in health and disease. J. Clin. Invest. 120, 3421-3431 (2010).

18. Tholen, M. et al. Out-of-frame start codons prevent translation of truncated nucleo-cytosolic cathepsin L in vivo. Nature 5, 4931 (2014).

19. Chen, J. M. et al. Cloning, isolation, and characterization of mammalian legumain, an asparaginyl endopeptidase. J. Biol. Chem. 272, 8090-8098 (1997).

20. Manoury, B. et al. An asparaginyl endopeptidase processes a microbial antigen for class II MHC presentation. Nature 396, 695-699 (1998).

21. Dall, E. \& Brandstetter, H. Mechanistic and structural studies on legumain explain its zymogenicity, distinct activation pathways, and regulation. Proc Natl Acad. Sci. USA 110, 10940-10945 (2013).

22. Maschalidi, S. et al. Asparagine endopeptidase controls anti-influenza virus immune responses through TLR7 activation. PLoS Pathog. 8, e1002841 (2012).

23. Watts, C., Matthews, S. P., Mazzeo, D., Manoury, B. \& Moss, C. X. Asparaginyl endopeptidase: case history of a class II MHC compartment protease. Immunol. Rev. 207, 218-228 (2005).

24. Liu, Z. et al. Neuroprotective actions of PIKE-L by inhibition of SET proteolytic degradation by asparagine endopeptidase. Mol. Cell 29, 665-678 (2008).

25. Zhang, Z. et al. Cleavage of tau by asparagine endopeptidase mediates the neurofibrillary pathology in Alzheimer's disease. Nat. Med. 20, 1254-1262 (2014).

26. Miller, G., Matthews, S. P., Reinheckel, T., Fleming, S. \& Watts, C. Asparagine endopeptidase is required for normal kidney physiology and homeostasis. FASEB J. 25, 1606-1617 (2011).

27. Chan, C.-B. et al. Mice lacking asparaginyl endopeptidase develop disorders resembling hemophagocytic syndrome. Proc. Natl Acad. Sci. USA 106, 468-473 (2009).

28. Shirahama-Noda, K. et al. Biosynthetic processing of cathepsins and lysosomal degradation are abolished in asparaginyl endopeptidase-deficient mice. J. Biol. Chem. 278, 33194-33199 (2003).

29. Krüger, M. et al. SILAC mouse for quantitative proteomics uncovers kindlin-3 as an essential factor for red blood cell function. Cell 134, 353-364 (2008).

30. Morita, Y. et al. Legumain/asparaginyl endopeptidase controls extracellular matrix remodeling through the degradation of fibronectin in mouse renal proximal tubular cells. FEBS Lett. 581, 1417-1424 (2007).

31. Loak, K. et al. Novel cell-permeable acyloxymethylketone inhibitors of asparaginyl endopeptidase. Biol. Chem. 384, 1239-1246 (2003).

32. Li, D. N., Matthews, S. P., Antoniou, A. N., Mazzeo, D. \& Watts, C. Multistep autoactivation of asparaginyl endopeptidase in vitro and in vivo. J. Biol. Chem. 278, 38980-38990 (2003).

33. Turvey, M. R., Wang, Y. \& Gu, Y. The effects of extracellular nucleotides on $[\mathrm{Ca} 2+]$ i signalling in a human-derived renal proximal tubular cell line (HKC8). J. Cell. Biochem. 109, 132-139 (2010).

34. Wang, W. et al. Up-regulation of lysosomal TRPML1 channels is essential for lysosomal adaptation to nutrient starvation. Proc. Natl Acad. Sci. USA 112, E1373-E1381 (2015). 
35. Lee, J. \& Bogyo, M. Synthesis and evalution of aza-peptidyl inhibitors of the lysosomal asparaginyl endopeptidase legumain. Bioorg. Med. Chem. Lett. 22, 1340-1343 (2012).

36. Zhong, Z., Wen, Z. \& Darnell, J. E. Stat3: a STAT family member activated by tyrosine phosphorylation in response to epidermal growth factor and interleukin-6. Science 264, 95-98 (1994).

37. Pang, M. et al. A novel STAT3 inhibitor, S3I-201, attenuates renal interstitial fibroblast activation and interstitial fibrosis in obstructive nephropathy. Kidney Int. 78, 257-268 (2010).

38. Hutchins, A. P. et al. Distinct transcriptional regulatory modules underlie STAT3's cell type-independent and cell type-specific functions. Nucleic Acids Res. 41, 2155-2170 (2013).

39. Bromberg, J. F. et al. Stat3 as an oncogene. Cell 98, 295-303 (1999).

40. Demaria, M. et al. STAT3 can serve as a hit in the process of malignant transformation of primary cells. Cell Death Differ. 19, 1390-1397 (2012).

41. Siveen, K. S. et al. Targeting the STAT3 signaling pathway in cancer: role of synthetic and natural inhibitors. Biochim. Biophys. Acta 1845, 136-154 (2014).

42. Carballo, M. et al. Oxidative stress triggers STAT3 tyrosine phosphorylation and nuclear translocation in human lymphocytes. J. Biol. Chem. 274, 17580-17586 (1999).

43. Vitner, E. B., Platt, F. M. \& Futerman, A. H. Common and uncommon pathogenic cascades in lysosomal storage diseases. J. Biol. Chem. 285, 20423-20427 (2010).

44. Barbieri, I. et al. Constitutively active Stat 3 enhances neu-mediated migration and metastasis in mammary tumors via upregulation of Cten. Cancer Res. 70 , $2558-2567$ (2010).

45. Stypmann, J. et al. Dilated cardiomyopathy in mice deficient for the lysosomal cysteine peptidase cathepsin L. Proc. Natl Acad. Sci. USA 99, 6234-6239 (2002).

46. Settembre, C. \& Ballabio, A. Lysosomal adaptation: how the lysosome responds to external cues. Cold Spring Harb. Perspect. Biol. 6, a016907 (2014).

47. Commisso, C. et al. Macropinocytosis of protein is an amino acid supply route in Ras-transformed cells. Nature 497, 633-637 (2013).

48. Palm, W. et al. The utilization of extracellular proteins as nutrients is suppressed by mTORC1. Cell 162, 259-270 (2015)

49. Mizushima, N. \& Komatsu, M. Autophagy: renovation of cells and tissues. Cell 147, 728-741 (2011).

50. Shen, S. et al. Cytoplasmic STAT3 represses autophagy by inhibiting PKR activity. Mol. Cell 48, 667-680 (2012).

51. Miao, L.-J. et al. Stat3 inhibits Beclin 1 expression through recruitment of HDAC3 in nonsmall cell lung cancer cells. Tumour Biol. 35, 7097-7103 (2014).

52. Pietrocola, F. et al. Regulation of autophagy by stress-responsive transcription factors. Semin. Cancer Biol. 23, 310-322 (2013).

53. Nielsen, R. et al. Increased lysosomal proteolysis counteracts protein accumulation in the proximal tubule during focal segmental glomerulosclerosis. Kidney Int. 84, 902-910 (2013).

54. Zoja, C., Benigni, A. \& Remuzzi, G. Protein overload activates proximal tubular cells to release vasoactive and inflammatory mediators. Exp. Nephrol. 7, 420-428 (1999).

55. Nakajima, H. et al. Activation of the signal transducer and activator of transcription signaling pathway in renal proximal tubular cells by albumin. J. Am. Soc. Nephrol. 15, 276-285 (2004).

56. Ivy, G. O., Schottler, F., Wenzel, J., Baudry, M. \& Lynch, G. Inhibitors of lysosomal enzymes: accumulation of lipofuscin-like dense bodies in the brain. Science 226, 985-987 (1984).

57. Höhn, A. \& Grune, T. Lipofuscin: formation, effects and role of macroautophagy. Redox Biol. 1, 140-144 (2013).

58. Aits, S. \& Jätttelä, M. Lysosomal cell death at a glance. J. Cell. Sci. 126 1905-1912 (2013).

59. Matthews, S. P. et al. Distinct protease requirements for antigen presentation in vitro and in vivo. J. Immunol. 184, 2423-2431 (2010).

60. Zaru, R., Ronkina, N., Gaestel, M., Arthur, J. S. C. \& Watts, C. The MAPKactivated kinase Rsk controls an acute Toll-like receptor signaling response in dendritic cells and is activated through two distinct pathways. Nat. Immunol. 8, 1227-1235 (2007).

61. Fox, C. et al. Inhibition of lysosomal protease cathepsin D reduces renal fibrosis in murine chronic kidney disease. Sci. Rep. 6, 20101 (2016).
62. Longo, P. A., Kavran, J. M., Kim, M.-S. \& Leahy, D. J. Transient mammalian cell transfection with polyethylenimine (PEI). Meth. Enzymol. 529, 227-240 (2013).

63. Schumm, K., Rocha, S., Caamano, J. \& Perkins, N. D. Regulation of p53 tumour suppressor target gene expression by the p52 NF25, 4820-4832 (2006).

64. Wiśniewski, J. R., Zougman, A., Nagaraj, N. \& Mann, M. Universal sample preparation method for proteome analysis. Nat. Meth. 6, 359-362 (2009).

65. Cox, J. \& Mann, M. MaxQuant enables high peptide identification rates, individualized p.p.b.-range mass accuracies and proteome-wide protein quantification. Nat. Biotechnol. 26, 1367-1372 (2008).

66. Cox, J. et al. Andromeda: a peptide search engine integrated into the MaxQuant environment. J. Proteome Res. 10, 1794-1805 (2011).

67. Shannon, P. et al. Cytoscape: a software environment for integrated models of biomolecular interaction networks. Genome Res.13, 2498-2504 (2003).

\section{Acknowledgements}

We thank Dr. Kamila Chughtai and Dr. Patrick Pedrioli for kindly monitoring SILAM labelling efficiency of the C57BL/6j 13C6-Lysine mouse colony and Rosie Clarke and Arlene Rennie for cell sorting. We are grateful to Prof. N.S. Sheerin for access to human kidney tissues, Matt Bogyo for epoxide and Michael acceptor AEP inhibitors, Doreen Keane for technical assistance, Kenny Milne for mass spectrometry, Thomas Macartney for CRISPR-Cas9 vector design and Steve Matthews and Krzys Wypijewski for comments on the manuscript. Generation and maintenance of protease-deficient mice was supported by SFB 850 project B7 and DFG grant RE1584/6-2 to T.R. V.P. was supported by AIRC Grant Number IG 16930. This work was funded by a Wellcome Trust Programme grant to C.W.

\section{Author contributions}

J.M.-F. designed, performed and analysed all experiments and wrote the paper. A.P. performed microscopy and helped with analysis of the micrographs. S.v.K. synthesised MVO26630. D.L.P. and I.M. provided tissues from SILAM mice. A.M. provided HKC-8 cells and primary human kidney epithelial cells. T.R. provided protease-deficient mice and tissues. V.P. provided STAT3c tissues and cells and other STAT3 reagents. C.W. helped with design and analysis of experiments, wrote the paper and generated funding.

\section{Additional information}

Supplementary Information accompanies this paper at https://doi.org/10.1038/s41467018-07741-6.

Competing interests: The authors declare no competing interests.

Reprints and permission information is available online at http://npg.nature.com/ reprintsandpermissions/

Publisher's note: Springer Nature remains neutral with regard to jurisdictional claims in published maps and institutional affiliations.

Open Access This article is licensed under a Creative Commons Attribution 4.0 International License, which permits use, sharing, adaptation, distribution and reproduction in any medium or format, as long as you give appropriate credit to the original author(s) and the source, provide a link to the Creative Commons license, and indicate if changes were made. The images or other third party material in this article are included in the article's Creative Commons license, unless indicated otherwise in a credit line to the material. If material is not included in the article's Creative Commons license and your intended use is not permitted by statutory regulation or exceeds the permitted use, you will need to obtain permission directly from the copyright holder. To view a copy of this license, visit http://creativecommons.org/ licenses/by/4.0/.

(c) The Author(s) 2018 